Gustavo Schvartsman

\title{
INCIDÊNCIA, PADRÕES DE PROGRESSÃO E DESFECHOS DE METÁSTASES CEREBRAIS DURANTE TRATAMENTO COM ANTI-PD-1 EM MELANOMA AVANÇADO
}

Tese apresentada à Faculdade Israelita de Ciências da Saúde Albert Einstein para obtenção do Título de Doutor em Ciências da Saúde.

São Paulo 
Gustavo Schvartsman

\section{INCIDÊNCIA, PADRÕES DE PROGRESSÃO E DESFECHOS DE METÁSTASES CEREBRAIS DURANTE TRATAMENTO COM ANTI-PD-1 EM MELANOMA AVANÇADO}

Tese apresentada à Faculdade Israelita de Ciências da Saúde Albert Einstein para obtenção do Título de Doutor em Ciências da Saúde.

Orientador: Prof. Dr. Nelson Hamerschlak

São Paulo

2019 
Schvartsman, Gustavo

Incidência, padrões de progressão e desfechos de metástases cerebrais durante tratamento com anti-PD-1 em melanoma avançado / Gustavo Schvartsman. -- São Paulo, 2019.

xiii, $41 \mathrm{f}$.

Tese (Doutorado) - Faculdade Israelita de Ciências da Saúde Albert Einstein. Instituto Israelita de Ensino e Pesquisa Albert Einstein. Programa de Pós-Graduação em Ciências da Saúde.

Título em inglês: Incidence, patterns of progression, and outcomes of brain metastases during treatment with anti-PD-1 for advanced metastatic melanoma.

1. Melanoma. 2. Inibidores de checkpoint imunológico. 3. Neoplasias encefálicas/secundário. 4. Resultado do tratamento.

NLM - WL358

Elaborada pelo Sistema Einstein Integrado de Bibliotecas 
FACULDADE ISRAELITA DE CIÊNCIAS DA SAÚDE ALBERT EINSTEIN

Coordenador do Curso de Pós-Graduação: Prof. Dr. Luiz Vicente Rizzo 
Gustavo Schvartsman

\section{INCIDÊNCIA, PADRÕES DE PROGRESSÃO E DESFECHOS DE METÁSTASES CEREBRAIS DURANTE TRATAMENTO COM ANTI-PD-1 EM MELANOMA AVANÇADO}

Presidente da banca: Prof. Dr. Nelson Hamerschlak

BANCA EXAMINADORA

Membros titulares:

Prof. Dr. Vicente Odone Filho

Prof. Dr. Sergio Daniel Simon

Prof. Dr. Jose Mauro Kutner

Membros suplentes:

Prof. Dr. Fernando Moura

Profa. Dra. Luciana Cavalheiro Marti

Aprovada em: 18/12/2019. 


\section{Dedicatória}

À memória do Prof. Dr. Samuel Schvartsman, meu avô, que dedicou sua vida ao progresso da ciência médica no Brasil e aos cuidados ao paciente. Sua vida acadêmica foi um exemplo a ser seguido, e seus valores foram transmitidos através das gerações. 


\section{Agradecimentos}

Primeiramente, ao meu orientador, Prof. Dr. Nelson Hamerschlak, por ter aceitado me orientar apesar da área diferente. Por ser uma inspiração, e por sua dedicação à toda minha família.

Aos meus pais, Benita e Claudio, por tudo que me ensinaram, pelo exemplo que até hoje me passam, e que tanto contribuíram para a realização do presente trabalho.

Ao meu irmão, Guilherme, por sempre elevar o debate ao maior nível, por nos manter atuais, ativos, e renovados, além do seu incessável apoio.

À Aline, com quem compartilho minha vida, e que está sempre ao meu lado, que me engrandece e me torna uma pessoa melhor.

A todos os professores com quem tive a oportunidade de aprender um pouco, que me estimularam e me desafiaram ao longo da minha vida e carreira.

A todos os meus amigos e colegas, de perto ou de longe, que me apoiaram e trilharam uma longa jornada comigo.

Ao Centro de Oncologia e Hematologia Família Dayan e Daycoval do Hospital Israelita Albert Einstein, como instituição e pelas pessoas que ali trabalham, que me deram oportunidades, que me apoiaram incondicionalmente e tornam possível o desenvolvimento científico em meio ao trabalho assistencial.

À University of Texas MD Anderson Cancer Center, em particular Dr. Hussein Tawbi e Dra. Isabella Glitza, pela mentoria e amizade, além da perseverança em manter colaborações científicas a 8 mil quilômetros de distância.

Por último, a todos os meus pacientes, pela confiança e carinho, e por quem constantemente busco melhorar suas condições e oferecer cuidados mais humanos. 
"Quem ensina o filho, ensina os filhos do filho." (Prof. Dr. Claudio Schvartsman, sobre seu pai)

Que o ciclo da vida permaneça girando assim. 


\section{Sumário}

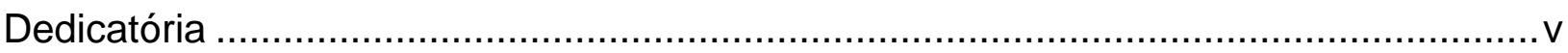

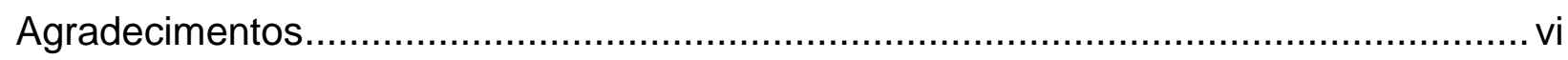

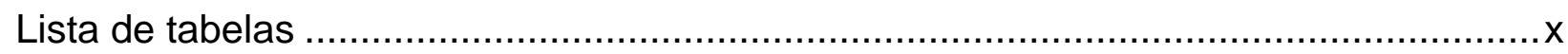

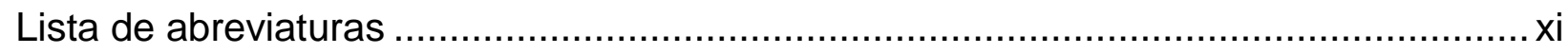

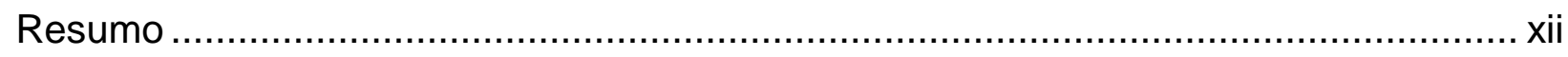

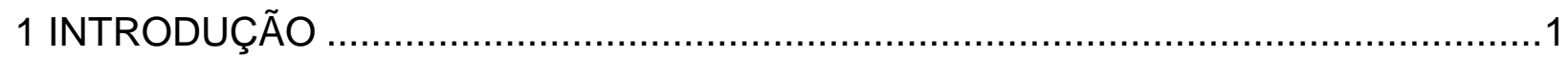

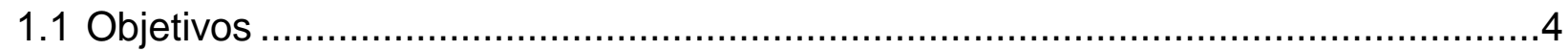

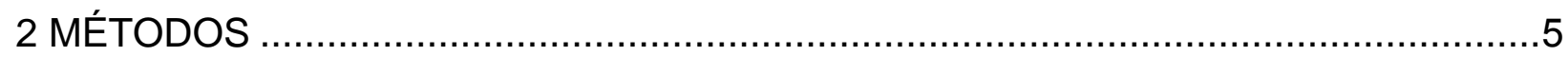

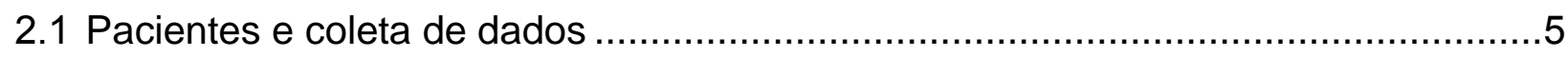

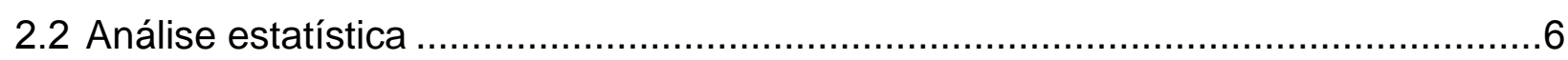

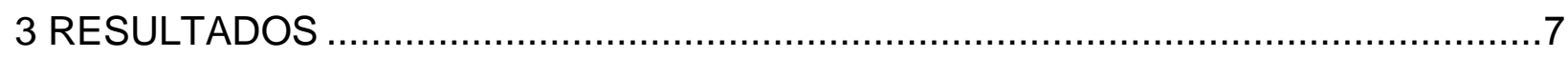

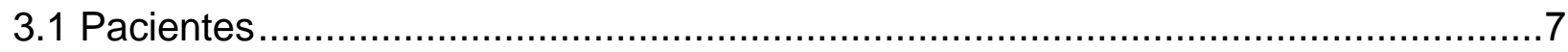

3.2 Grupo "Sem Metástase Cerebral por Melanoma Prévia" ...................................11

3.3 Grupo "Com Metástase Cerebral por Melanoma Prévia"..................................20

3.4 Radionecrose durante o tratamento com anti-PD-1 .....................................23

3.5 Tratamento de metástase cerebral por melanoma ........................................23

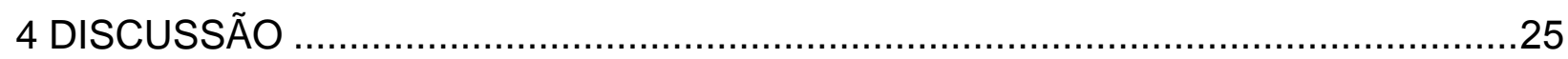

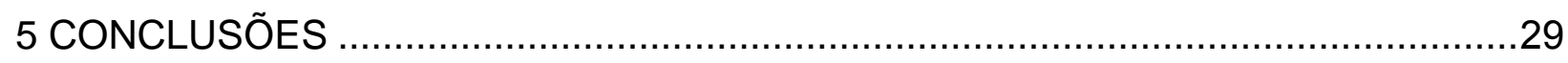

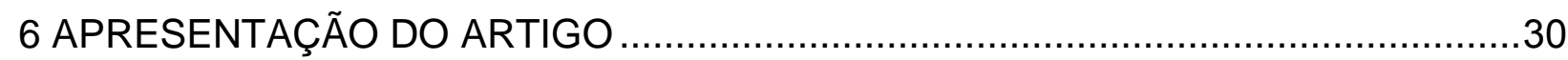

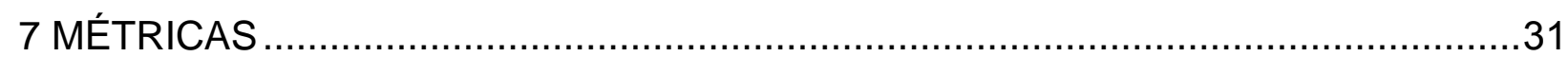

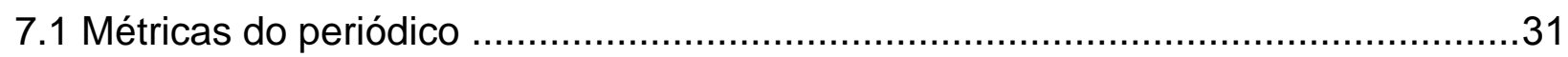

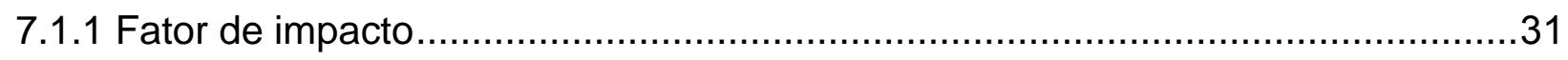

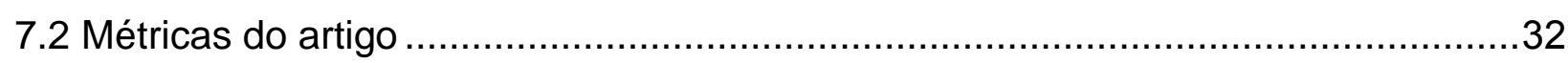

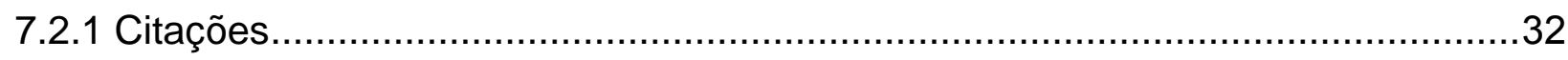

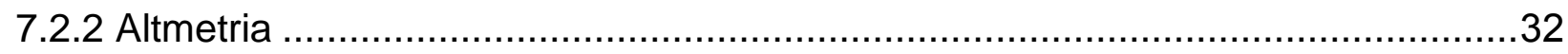

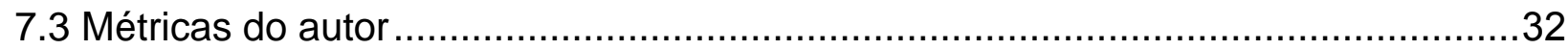

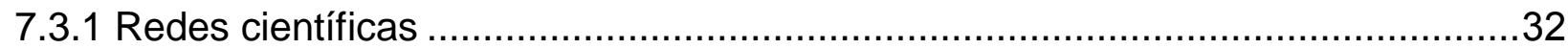

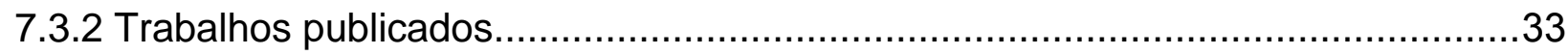

7.3.3 Mediana do fator de impacto dos trabalhos publicados.................................35

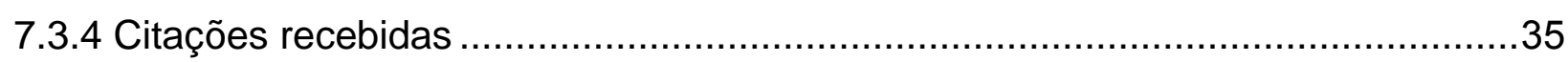

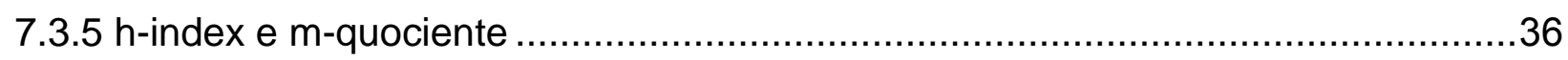


8 REFERÊNCIAS.

.38

Abstract 


\section{Lista de tabelas}

Tabela 1. Características basais ao início do anti-PD-1 ....................................

Tabela 2. Características basais de pacientes do grupo "Sem Metástase Cerebral por Melanoma Prévia", de acordo com momento do diagnóstico .................................9

Tabela 3. Análise uni/multivariada de fatores prognósticos para sobrevida livre de metástase cerebral ................................................................................. 14

Tabela 4. Análise uni/multivariada de fatores prognósticos associados a sobrevida global em pacientes do grupo "Sem Metástase Cerebral por Melanoma Prévia". 16

Tabela 5. Análise uni/multivariada de fatores prognósticos associados a sobrevida global para todos os pacientes

Tabela 6. Análise univariada de fatores prognósticos associados com SNC-SLP e SISSLP entre pacientes do grupo "Com Metástase Cerebral por Melanoma Prévia" 21

Tabela 7. Resumo do tratamento local direcionado ao cérebro .24 


\section{Lista de abreviaturas}

CTLA-4 Cytotoxic T-Lymphocyte Antigen-4

HR Hazard ratio (razão de risco)

IC Intervalo de confiança

IIQ Intervalo interquartil

IMC Índice de massa corpórea

LDH Lactato desidrogenase

LITT Terapia intersticial por laser (Laser Interstitial Thermal Therapy)

MCM Metástase cerebral por melanoma

MDACC The University of Texas MD Anderson Cancer Center

PD-1 Programmed-death 1

PET-TC Tomografia por emissão de pósitrons (Positron Emission Tomography Computed Tomography)

RM Ressonância magnética

RN Radionecrose

SG Sobrevida global

SIS Sistêmica

SLMC Sobrevida livre de metástase cerebral

SLP Sobrevida livre de progressão

SNC Sistema nervoso central

SRS Radiocirurgia estereotáxica (Stereotactic radiosurgery)

TC Tomografia computadorizada 


\section{Resumo}

Introdução: As metástases cerebrais por melanoma ocorrem em até $50 \%$ dos pacientes com melanoma metastático e representam um local frequente de falha de tratamento sistêmico. A imunoterapia revolucionou o tratamento do melanoma, e demonstrou eficácia em pacientes com metástases cerebrais. No entanto, pouco se sabe sobre a incidência de novas metástases cerebrais por melanoma em pacientes sem diagnóstico prévio, padrões de progressão com o tratamento e desfechos de longo prazo em pacientes com e sem metástases cerebrais tratados com imunoterapia à base de anti-PD-1. Objetivos: Avaliar a incidência de metástases cerebrais por melanoma em pacientes sem diagnóstico prévio ao início do tratamento com anti-PD-1; avaliar os padrões de progressão de pacientes com e sem metástases cerebrais por melanoma ao início do tratamento com anti-PD-1; e avaliar os desfechos de longo prazo nas diferentes populações na atual era de imunoterapia e tratamento intensivo local de metástases cerebrais. Métodos: Trezentos e vinte pacientes com melanoma metastático tratados com anti-PD-1 no MD Anderson Cancer Center foram revisados. Análises foram realizadas para identificar fatores associados à sobrevida livre de metástases cerebrais e sobrevida global usando modelos de regressão de Cox e o método de Kaplan-Meier. Resultados: A idade mediana foi de 63,3 anos. A incidência de novas metástases cerebrais por melanoma entre os pacientes sem diagnóstico prévio foi de 27,3\% ( $\mathrm{N}=67)$. Destes, 21 (8,6\%) desenvolveram metástases cerebrais durante o tratamento com anti-PD-1, dos quais 12 (4,9\%) apresentaram apenas progressão no sistema nervoso central. Entre os pacientes com metástases cerebrais pré-existentes, 15 (20,0\%) progrediram apenas no cérebro e 19 (25,3\%) progrediram intra e extracranialmente; até o fechamento dos dados, 27 (36,0\%) não haviam progredido. A sobrevida global a partir do início do anti-PD-1 não foi significativamente diferente entre pacientes com ou sem metástase cerebral prévia ao uso de anti-PD-1 (valor- $\mathrm{p}=0,359)$, mas desenvolver metástases cerebrais durante ou após o anti-PD-1 foi associado com pior sobrevida global ( $\mathrm{HR}=4,70,95 \% \mathrm{Cl}: 3,18-6,93)$. Radionecrose ocorreu em 11,3\% (7 de 62) dos pacientes no grupo com metástase cerebral prévia e que receberam radiocirurgia estereotáxica. Conclusões: A terapia com anti-PD-1 pode alterar a história natural de pacientes com metástase cerebral por melanoma préexistente, apresentando desfechos semelhantes em pacientes com ou sem doença 
intracraniana antes do início do tratamento. No entanto, a falha do anti-PD-1 no sistema nervoso central é frequente e ainda é um fator de pior prognóstico. Nossos resultados reforçam a atividade do anti-PD-1 em pacientes com metástases cerebrais por melanoma, porém os achados sugerem que a obtenção rotineira de imagens do crânio seja recomendada, mesmo na ausência de sintomas neurológicos.

Descritores: Melanoma; Inibidores de checkpoint imunológico; Neoplasias encefálicas/secundário; Resultado do tratamento 


\section{INTRODUÇÃO}

O melanoma representa um dos tumores de pele com maior agressividade. Ocorre frequentemente em população caucasiana, sendo o principal fator de risco a radiação ultravioleta. ${ }^{(1)}$ No mundo, o melanoma representa a terceira neoplasia mais prevalente em homens, e a quinta em mulheres. ${ }^{(2)}$ No Brasil, estima-se mais de 6000 casos novos no ano de 2018 e 1700 mortes pela doença. ${ }^{(3)} \mathrm{A}$ ampla maioria dos casos se apresenta como doença localizada ao diagnóstico, com taxas de cura elevadas. Porém, em até $20 \%$ dos casos, o tumor apresenta invasão regional e metástases a distância, contexto associado à taxas de sobrevida substancialmente reduzidas. ${ }^{(2)}$

O cenário do tratamento da doença metastática mudou radicalmente na década de $2010 .{ }^{(4)}$ A identificação da mutação oncogênica na porção V600 do gene BRAF, que ocorre em cerca de metade dos casos, e o subsequente desenvolvimento de pequenas moléculas inibidoras da proteína BRAF trouxeram grande benefício para essa população. ${ }^{(5)}$ Dados de 5 anos da combinação de dabrafenibe, um inibidor de BRAF, associado a trametinibe, um inibidor de MEK (proteína downstream na cascata de sinalização, que funciona como mecanismo de escape do bloqueio isolado da proteína BRAF) resultou em uma taxa de sobrevida global de $34 \%$ em 5 anos. ${ }^{(6)}$ Historicamente, esse número era inferior a 15\%. Hoje, já são três combinações diferentes aprovadas ao redor do mundo (inibidores de BRAF e MEK, respectivamente: vemurafenibe e cobimetinibe; dabrafenibe e trametinibe; encorafenibe e binimetinibe), sendo que as duas primeiras estão disponíveis para uso no Brasil.

Uma outra opção de tratamento descoberta simultaneamente é a imunoterapia. O tratamento consiste em anticorpos monoclonais contra proteínas que inibem a atuação do sistema imune, chamadas de checkpoints. Ao serem bloqueadas, o sistema imune pode voltar a exercer sua atividade. ${ }^{(7)} \mathrm{O}$ melanoma é um tumor particularmente sensível à imunoterapia por sua alta carga mutacional, decorrente do extenso dano ao DNA do melanócito, o que gera múltiplos neoantígenos (proteínas aberrantes) e um maior potencial de reconhecimento pelo sistema imune. ${ }^{(8)}$ A primeira molécula aprovada para uso, o ipilimumabe, inibe a proteína Cytotoxic T-Lymphocyte Antigen-4 (CTLA-4). Resultados de estudos com quase 10 anos de seguimento demonstram sobrevida de longo prazo em $20 \%$ dos pacientes, após apenas 4 doses de ipilimumabe administradas a cada 3 semanas. ${ }^{(9)}$ Porém, o mecanismo de inibição não é 
específico para a via de escape tumoral, de forma que a toxicidade imuno-mediada dessa medicação é significativa, com cerca de $40 \%$ dos pacientes apresentando eventos adversos graves. ${ }^{(10)}$

A segunda e mais importante classe de tratamento imunoterápico que ganhou aprovação pelas agências regulatórias foi a de inibidores do programmed death-1 (PD-1). Essa proteína é mais específica a mecanismos de escape tumoral, de forma que seus resultados de eficácia foram mais significativos, resultando em taxa de sobrevida em 5 anos de até $41 \%$ em pacientes sem tratamento prévio, com toxicidades importantes em cerca de $15 \%$ dos casos. ${ }^{(11)}$ Dois medicamentos são aprovados para uso em diversos países, incluindo o Brasil: pembrolizumabe e nivolumabe, sendo que o último também é aprovado para uso em combinação com ipilimumabe. ${ }^{(12)}$

Apesar dos bons resultados obtidos recentemente, mais de metade dos pacientes ainda vem a falecer do melanoma metastático. Uma das principais causas de falha do tratamento e de óbito é a disseminação ao sistema nervoso central (SNC). O melanoma possui a maior propensão de invadir o SNC, ficando atrás apenas dos cânceres de pulmão e de mama em incidência absoluta de metástases cerebrais (pelo elevado número de casos de ambos). ${ }^{(13)}$ Aproximadamente $40-50 \%$ dos pacientes com melanoma em estágio IV desenvolvem metástases cerebrais por melanoma (MCM) clinicamente detectáveis, números que chegam até $73 \%$ em séries de autópsias. ${ }^{(14,15)}$ Historicamente, o prognóstico nesta população é ruim e a doença do SNC é a principal causa de morte. ${ }^{(16)}$

A terapia sistêmica tradicionalmente não é considerada uma abordagem eficaz para as MCM, uma vez que a maioria dos agentes quimioterápicos não penetra na barreira hematoencefálica. Temozolomida, um agente quimioterápico alquilante com atividade contra o melanoma e penetração do SNC, demonstrou atividade intracraniana limitada, com uma taxa de resposta de apenas cerca de $7 \% .{ }^{(17)}$

A ampla maioria dos estudos com imunoterapia e terapia alvodirigida contra BRAF/MEK excluiu sistematicamente a população com MCM ativa (sem tratamento com radioterapia ou cirurgia). Recentemente, o uso desses agentes foi testado em estudos clínicos com pacientes especificamente com metástases ativas no SNC para avaliação de segurança e eficácia. A terapia alvo-dirigida utilizando inibidores de BRAF como agente único, ou terapia combinada com inibidores de MEK, em pacientes com melanoma BRAF V600 mutado, produziu altas taxas de resposta intracranianas, que foram concordantes com as respostas extracranianas. ${ }^{(18-21)}$ Apesar 
da boa penetração no cérebro, a sobrevida livre de progressão (SLP) foi significativamente menor do que seria esperado para pacientes com metástases extracranianas apenas (6 versus 12 meses), sendo que metade das falhas do tratamento foram apenas no cérebro. ${ }^{(21)}$

Embora os anticorpos monoclonais utilizados na imunoterapia sejam moléculas grandes e com baixa penetração no SNC, as células T e outras células do sistema imune têm acesso facilitado através da membrana basal do parênquima cerebral. $^{(22)}$ A atuação de citocinas no compartimento perivascular e enzimas secretadas por macrófagos induzem a quebra de ligações entre astrócitos e a matriz extracelular. ${ }^{(23,24)} \mathrm{A}$ dissolução dessa matriz permite um livre fluxo de células $T$ para 0 parênquima cerebral. Ademais, novas descobertas sobre a ramificação do sistema linfático de drenagem intracraniana, até pouco tempo atrás considerado escasso, gera a hipótese de uma rota alternativa para interação entre o melanócito e linfócitos $T$ sentinelas. ${ }^{(25)}$ No contexto clínico, o bloqueio de ambos CTLA-4 e PD-1 foi realizado em pacientes com MCM não tratada. O ipilimumabe levou a uma taxa de resposta de $16 \%$ em pacientes assintomáticos com MCM, semelhante à taxa de resposta extracraniana. ${ }^{(26)}$ Porém, uma coorte de pacientes recebendo esteroides para controlar os sintomas decorrentes de edema cerebral tiveram desempenho insatisfatório, tanto pela doença mais avançada, quanto pelo efeito imunossupressor do corticoide. ${ }^{(26)} \mathrm{O}$ pembrolizumabe, um anti-PD-1, resultou em uma taxa de resposta de $22 \%$ intra e extracranialmente em uma pequena coorte de pacientes assintomáticos. ${ }^{(27)} \mathrm{O}$ estudo CheckMate-204 ( $\mathrm{N}=101)$ utilizou o anti-CTLA-4 ipilimumabe em combinação com o anti-PD-1 nivolumabe por 4 doses, seguido de manutenção com nivolumabe isolado para pacientes previamente não tratados com pelo menos uma MCM ativa, de tamanho entre 0,5 e $3,0 \mathrm{~cm}$ e sem sintomas neurológicos ou uso de esteróides. ${ }^{(28)} \mathrm{A}$ taxa de resposta intracraniana foi de $55 \%$ e a taxa de resposta completa foi de $26 \%$, com respostas intracranianas e extracranianas amplamente concordantes. É importante ressaltar que, até o momento da apresentação dos dados, a duração da resposta e a SLP mediana não haviam sido atingidas para os pacientes que tiveram resposta. A sobrevida global (SG) em 18 meses foi de $75 \%$, número sem precedentes nessa população de alto risco e sem tratamento local de SNC com radioterapia obrigatório. Uma segunda coorte do estudo, porém, analisou 18 pacientes com sintomas ou uso de corticoterapia para controle de edema perilesional. $O$ resultado com o mesmo tratamento foi significativamente inferior, com taxa de resposta de $22 \%$ e SG de $66 \%$ 
em 6 meses. ${ }^{(28)}$ Outro ensaio clínico (ABC Study) também avaliou a combinação de nivolumabe e ipilimumabe versus nivolumabe isolado $(\mathrm{N}=63)$ em uma população semelhante à coorte assintomática do estudo anterior. Pacientes com MCM apresentaram taxa de resposta intracraniana de $46 \%$ com a combinação e $20 \%$ com nivolumabe isolado. ${ }^{(29)}$ Ambos os estudos sugerem que a imunoterapia pode fornecer respostas duráveis não apenas para pacientes com doença extracraniana, mas também para pacientes com MCM.

Embora esses resultados sejam encorajadores, os estudos clínicos prospectivos direcionados à população com MCM representam apenas $10 \%$ dos pacientes recrutados para todos os estudos de melanoma metastático, número bastante inferior à incidência de lesões cerebrais causados pela doença. ${ }^{\left({ }^{(30)}\right.}$ Ainda, os dados são escassos em relação à incidência de novas MCM durante e após tratamento com anti-PD-1, aos padrões de progressão de doença (intra versus extracranialmente) e o impacto de MCM sobre desfechos de longo prazo na era moderna da imunoterapia e tratamento intensivo local com radioterapia. Assim, revisamos os registros de pacientes tratados com anti-PD-1 na University of Texas MD Anderson Cancer Center (MDACC) para responder essas questões.

\subsection{Objetivos}

1. Avaliar a incidência de metástase cerebral por melanoma em pacientes sem diagnóstico prévio ao início do tratamento com anti-PD-1;

2. Avaliar os padrões de progressão de pacientes com e sem metástase cerebral por melanoma ao início do tratamento com anti-PD-1;

3. Avaliar os desfechos de longo prazo (sobrevida livre de progressão e sobrevida global) nas diferentes populações na atual era de imunoterapia e tratamento intensivo local de sistema nervoso central. 
2 MÉTODOS

\subsection{Pacientes e coleta de dados}

De acordo com um protocolo aprovado pelo Institutional Review Board do MDACC e pelo Comitê de Ética do Hospital Israelita Albert Einstein (CAAE: 76229517.1.0000.0071), os registros de pacientes tratados no MDACC com melanoma avançado que receberam agente único com anti-PD-1 (nivolumabe ou pembrolizumabe) de janeiro de 2012 a julho de 2016 foram revisados. Um mínimo de 6 meses de seguimento clínico foi instituído para inclusão nesta análise, a fim de excluir os pacientes vistos apenas como uma consulta de segunda opinião e, portanto, sem dados de tratamento. Nenhum paciente com progressão precoce da doença foi excluído. Dados demográficos do paciente, características do tumor primário, resultados de testes clínicos moleculares, linhas de tratamento no contexto metastático recebidos antes do diagnóstico de $\mathrm{MCM}$ e os resultados do tratamento foram coletados.

Os pacientes foram classificados em dois grupos principais: aqueles que tinham evidência de MCM antes do início do anti-PD-1 ("Com MCM Prévia"); e aqueles que não tinham evidência de MCM ("Sem MCM Prévia"). Neste último, os pacientes que desenvolveram MCM durante o tratamento anti-PD-1, ou dentro de 30 dias após a descontinuação do anti-PD-1, foram categorizados como "Durante Anti-PD1" e pacientes diagnosticados com MCM mais de 30 dias após a descontinuação do anti-PD-1 foram categorizados como "Após Anti-PD-1". O diagnóstico de MCM foi baseado em ressonâncias magnéticas (RM) cerebrais seriadas, ou tomografias computadorizadas (TC) de crânio com contraste endovenoso, quando a RM estava contraindicada. Apenas pacientes com imagem do SNC dentro de 3-4 semanas antes e após a última dose de anti-PD-1 foram incluídos nesta análise, a fim de determinar com precisão se a doença do SNC estava presente no início e na interrupção do anti-PD-1. A doença sistêmica (SIS) foi monitorada com exames de imagem de corpo (TC de tórax, abdome e pelve, ou tomografia por emissão de pósitrons [PET-TC]). A radionecrose, um fator de confusão importante de mudança de aspecto na imagem após radioterapia intracraniana, foi definida com base na patologia, quando houve ressecção cirúrgica (alterações reativas e necrose, sem células tumorais viáveis), ou 
com base nas características radiológicas convencionais naqueles que não realizaram cirurgia (realce periférico e hipointensidade central), como descrito anteriormente. ${ }^{(31)}$

\subsection{Análise estatística}

As características dos pacientes foram resumidas com porcentagens ou medianas e amplitudes ou intervalo interquartil (IIQ), ou com médias e desvios padrão. Os valores de linha de base foram comparadas entre os diferentes grupos pelo teste exato de Fisher e pelo teste de Kruskal-Wallis para variáveis categóricas e contínuas, respectivamente. O tempo de SG foi calculado a partir da data de início do anti-PD-1 até a última data vital. O tempo de SLP intracraniana e extracraniana (SNC/SIS-SLP) foram calculados a partir da data de início do anti-PD-1 até o momento do desenvolvimento de MCM nova/progressiva (SNC-SLP) ou progressão sistêmica da doença (SIS-SLP), ou morte como evento para ambas as análises. O tempo de sobrevida livre de metástases cerebrais (SLMC) foi calculado a partir da data de início do anti-PD-1 até a data do desenvolvimento de MCM ou morte, no grupo "Sem MCM Prévia". Os pacientes que estavam vivos e não desenvolveram MCM nova/progressiva, ou progressão de doença sistêmica até a última data de imagem disponível foram censurados. As SNC-SLP e SIS-SLP foram definidas no grupos "Com MCM Prévia", enquanto que a SLMC foi definido no grupo "Sem MCM Prévia". As distribuições de curvas de SG, SLMC e SNC-SLP/SIS-SLP foram estimadas usando o método de Kaplan-Meier e comparadas entre os grupos com o teste log-rank. As associações entre as variáveis clínicas, o desenvolvimento de MCM e o tempo até eventos foram avaliados usando o modelo de regressão de Cox. A associação de desenvolvimento de MCM e morte foi tratada de maneira interdependente ao longo do tempo, como variável covariante. Variáveis com valores-p menores que 0,1 na análise univariada foram selecionadas para análise multivariada, exceto para variáveis que reduziram substancialmente o tamanho da amostra se incluídas. Esse valor de corte foi selecionado para garantir que variáveis relevantes sejam ajustadas na análise multivariada. Todas as análises estatísticas foram realizadas usando os softwares $R$ versão 3.5.1 e o SAS 9.4. Todos os testes estatísticos utilizaram um nível de significância de $5 \%$ e não foram feitos ajustes para testes múltiplos. 


\section{RESULTADOS}

\subsection{Pacientes}

Identificamos 320 pacientes com melanoma metastático que foram tratados com anti-PD-1 e acompanhados por pelo menos 6 meses com imagens seriadas do corpo e do SNC. As características basais dos pacientes são apresentadas na tabela 1.

Tabela 1. Características basais ao início do anti-PD-1

\begin{tabular}{|c|c|c|c|c|c|}
\hline Variável & $\mathbf{n}$ & Total & $\begin{array}{c}\text { Sem MCM } \\
\text { prévia }\end{array}$ & $\begin{array}{c}\text { Com MCM } \\
\text { prévia }\end{array}$ & Valor-p \\
\hline Idade, M (DP) & 320 & $61,3(13,6)$ & $62,6(13,4)$ & $57,0(13,5)$ & 0,002 \\
\hline Gênero, n(\%) & 320 & & & & 0,323 \\
\hline Feminino & & $103(32,2)$ & $75(30,6)$ & $28(37,3)$ & \\
\hline Masculino & & $217(67,8)$ & $170(69,4)$ & $47(62,7)$ & \\
\hline Raça, n(\%) & 317 & & & & $<0,001$ \\
\hline Outro & & $23(7,3)$ & $19(7,9)$ & $4(5,3)$ & \\
\hline Branco & & $294(92,7)$ & $223(92,1)$ & $71(94,7)$ & \\
\hline Patologia, $\mathrm{n}(\%)$ & 320 & & & & 0,136 \\
\hline Cutâneo, não-acral & & $190(59,4)$ & $146(59,6)$ & $44(58,7)$ & \\
\hline Cutâneo, acral & & $6(1,9)$ & $4(1,6)$ & $2(2,7)$ & \\
\hline Indeterminado & & $84(26,2)$ & $59(24,1)$ & $25(33,3)$ & \\
\hline Mucoso & & $22(6,9)$ & $19(7,8)$ & $3(4,0)$ & \\
\hline Uveal & & $18(5,6)$ & $17(6,9)$ & $1(1,3)$ & \\
\hline Ulceração, n(\%) & 180 & & & & 0,713 \\
\hline Ausente & & $91(50,6)$ & $71(49,7)$ & $20(54,1)$ & \\
\hline Presente & & $89(49,4)$ & $72(50,3)$ & $17(45,9)$ & \\
\hline LDH, n(\%) & 320 & & & & 0,085 \\
\hline Não elevado & & $149(46,6)$ & $121(49,4)$ & $28(37,3)$ & \\
\hline Elevado & & $171(53,4)$ & $124(50,6)$ & $47(62,7)$ & \\
\hline Localização, n(\%) & 255 & & & & 0,503 \\
\hline Não-cabeça e pescoço & & $172(67,5)$ & $136(66,3)$ & $36(72,0)$ & \\
\hline Cabeça e pescoço & & $83(32,5)$ & $69(33,7)$ & $14(28,0)$ & \\
\hline IMC, $n(\%)$ & 278 & & & & 0,095 \\
\hline$<18$ & & $1(0,4)$ & $0(0,0)$ & $1(1,6)$ & \\
\hline$\geq 18, \leq 25$ & & $63(22,7)$ & $44(20,4)$ & $19(30,6)$ & \\
\hline$>25, \leq 30$ & & $98(35,3)$ & $78(36,1)$ & $20(32,3)$ & \\
\hline$>30$ & & $116(41,7)$ & $94(43,5)$ & $22(35,5)$ & \\
\hline BRAF, n(\%) & 298 & & & & 0,052 \\
\hline Sem mutação & & $191(64,1)$ & $153(68,0)$ & $38(52,1)$ & \\
\hline Outra mutação & & $22(7,4)$ & $17(7,6)$ & $5(6,8)$ & \\
\hline V600E & & $70(23,5)$ & $45(20,0)$ & $25(34,2)$ & \\
\hline
\end{tabular}




\begin{tabular}{|c|c|c|c|c|c|}
\hline V600K & & $15(5,0)$ & $10(4,4)$ & $5(6,8)$ & \\
\hline NRAS, $\mathrm{n}(\%)$ & 277 & & & & 0,132 \\
\hline Sem mutação & & $211(76,2)$ & $167(78,4)$ & $44(68,8)$ & \\
\hline Mutação & & $66(23,8)$ & $46(21,6)$ & $20(31,2)$ & \\
\hline $\mathrm{KIT}, \mathrm{n}(\%)$ & 279 & & & & 0,678 \\
\hline Sem mutação & & $242(86,7)$ & $186(87,3)$ & $56(84,8)$ & \\
\hline Mutação & & $37(13,3)$ & $27(12,7)$ & $10(15,2)$ & \\
\hline PTEN, $n(\%)$ & 67 & & & & 1,000 \\
\hline Mutação & & $10(14,9)$ & $7(15,2)$ & $3(14,3)$ & \\
\hline Sem mutação & & $57(85,1)$ & $39(84,8)$ & $18(85,7)$ & \\
\hline Estágio, n(\%) & 320 & & & & 0,003 \\
\hline Estágio III & & $22(6,9)$ & $22(9,0)$ & $0(0,0)$ & \\
\hline Estágio IV & & $298(93,1)$ & $223(91,0)$ & $75(100,0)$ & \\
\hline Metástase linfonodal, $n(\%)$ & 320 & & & & 0,658 \\
\hline Não & & $87(27,2)$ & $65(26,5)$ & $22(29,3)$ & \\
\hline Sim & & $233(72,8)$ & $180(73,5)$ & $53(70,7)$ & \\
\hline Metástase hepática, n(\%) & 320 & & & & 0,174 \\
\hline Não & & $201(62,8)$ & $159(64,9)$ & $42(56,0)$ & \\
\hline Sim & & $119(37,2)$ & $86(35,1)$ & $33(44,0)$ & \\
\hline Metástase pulmonar, n(\%) & 320 & & & & 0,102 \\
\hline Não & & $121(37,8)$ & $99(40,4)$ & $22(29,3)$ & \\
\hline $\operatorname{Sim}$ & & $199(62,2)$ & $146(59,6)$ & $53(70,7)$ & \\
\hline Metástase óssea, n(\%) & 320 & & & & 0,756 \\
\hline Não & & $247(77,2)$ & $190(77,6)$ & $57(76,0)$ & \\
\hline $\operatorname{Sim}$ & & $73(22,8)$ & $55(22,4)$ & $18(24,0)$ & \\
\hline $\begin{array}{l}\text { Outras metástases abdominais } \\
\mathrm{n}(\%)\end{array}$ & 320 & & & & 0,002 \\
\hline Não & & $215(67,2)$ & $176(71,8)$ & $39(52,0)$ & \\
\hline $\operatorname{Sim}$ & & $105(32,8)$ & $69(28,2)$ & $36(48,0)$ & \\
\hline Anti, BRAF prévio, n(\%) & 320 & & & & 0,013 \\
\hline Não & & $244(76,2)$ & $195(79,6)$ & $49(65,3)$ & \\
\hline $\operatorname{Sim}$ & & $76(23,8)$ & $50(20,4)$ & $26(34,7)$ & \\
\hline Ipilimumabe prévio, $\mathrm{n}(\%)$ & 320 & & & & 1,000 \\
\hline Não & & $125(39,1)$ & $96(39,2)$ & $29(38,7)$ & \\
\hline Sim & & $195(60,9)$ & $149(60,8)$ & $46(61,3)$ & \\
\hline $\begin{array}{l}\text { Quimioterapia prévia } \\
\text { (Temozolamida), } \mathrm{n}(\%)\end{array}$ & 320 & & & & 0,012 \\
\hline Não & & $246(76,9)$ & $197(80,4)$ & $49(65,3)$ & \\
\hline $\operatorname{Sim}$ & & $74(23,1)$ & $48(19,6)$ & $26(34,7)$ & \\
\hline $\begin{array}{l}\text { Quimioterapia prévia (não- } \\
\text { temozolamida*), } n(\%)\end{array}$ & 320 & & & & 0,289 \\
\hline Não & & $178(55,6)$ & $132(53,9)$ & $46(61,3)$ & \\
\hline $\operatorname{Sim}$ & & $142(44,4)$ & $113(46,1)$ & $29(38,7)$ & \\
\hline
\end{tabular}

${ }^{*}$ Quimioterapia não-temozolomida incluiu carbo/cisplatina, paclitaxel, nab-paclitaxel, dacarbazina, vinblastina, carmustina. M: média; DP: desvio padrão; MCM: metástase cerebral por melanoma; LDH: lactato desidrogenase; IMC: índice de massa corpórea; N/A: não-aplicável. 
A idade mediana dos pacientes foi de 63,3 anos (amplitude: 16,5 90,6 anos) e 217 (67,8\%) eram do sexo masculino. Os níveis de lactato desidrogenase (LDH) acima do limite superior de referência foram observados em mais da metade dos pacientes $(53,4 \%, N=171)$. Os dados da análise de mutação para o teste BRAF estavam disponíveis para 298 pacientes, com $85(28,5 \%)$ deles portadores de mutação BRAF V600E ( $\mathrm{N}=70)$ ou BRAF V600K ( $\mathrm{N}=15)$. Mutação no gene NRAS foi observada em 66 pacientes $(23,8 \%$ de 277 pacientes avaliáveis). Terapias sistêmicas prévias no cenário metastático incluíram terapia alvo-dirigida (23,8\%), ipilimumabe $(60,9 \%)$ e quimioterapia $(67,5 \%)$, com $23,1 \%$ de temozolomida e $44,4 \%$ de regimes nãotemozolomida (incluindo carbo/cisplatina, paclitaxel, nab-paclitaxel, dacarbazina, vinblastina e carmustina). As características detalhadas dos subgrupos "Durante AntiPD-1" e "Após Anti-PD-1" são exibidas na tabela 2.

Tabela 2. Características basais de pacientes do grupo "Sem Metástase Cerebral por Melanoma Prévia", de acordo com momento do diagnóstico

\begin{tabular}{|c|c|c|c|c|c|c|}
\hline Variável & $\mathbf{n}$ & Total & Após Anti-PD-1 & $\begin{array}{c}\text { Durante } \\
\text { Anti-PD-1 }\end{array}$ & Sem MCM & Valor-p \\
\hline Idade, M (DP) & 245 & $62,6(13,4)$ & $60,4(13,7)$ & $53,2(15,7)$ & $64,3(12,5)$ & $<0,001$ \\
\hline Gênero, n(\%) & 245 & & & & & 0,186 \\
\hline Feminino & & $75(30,6)$ & $17(37,0)$ & $9(42,9)$ & $49(27,5)$ & \\
\hline Masculino & & $170(69,4)$ & $29(63,0)$ & $12(57,1)$ & $129(72,5)$ & \\
\hline Raça, $n(\%)$ & 242 & & & & & 0,354 \\
\hline Outro & & $19(7,9)$ & $6(13,0)$ & $1(5,3)$ & $12(6,8)$ & \\
\hline Branco & & $223(92,1)$ & $40(87,0)$ & $18(94,7)$ & $165(93,2)$ & \\
\hline Patologia, $\mathrm{n}(\%)$ & 245 & & & & & 0,935 \\
\hline Cutâneo, não-acral & & $146(59,6)$ & $27(58,7)$ & $13(61,9)$ & $106(59,6)$ & \\
\hline Cutâneo, acral & & $4(1,6)$ & $1(2,2)$ & $0(0,0)$ & $3(1,7)$ & \\
\hline Indeterminado & & $59(24,1)$ & $13(28,3)$ & $4(19,0)$ & $42(23,6)$ & \\
\hline Mucoso & & $19(7,8)$ & $3(6,5)$ & $3(14,3)$ & $13(7,3)$ & \\
\hline Uveal & & $17(6,9)$ & $2(4,3)$ & $1(4,8)$ & $14(7,9)$ & \\
\hline Ulceração, n(\%) & 143 & & & & & 0,098 \\
\hline Ausente & & $71(49,7)$ & $17(68,0)$ & $8(53,3)$ & $46(44,7)$ & \\
\hline Presente & & $72(50,3)$ & $8(32,0)$ & $7(46,7)$ & $57(55,3)$ & \\
\hline LDH, n(\%) & 245 & & & & & 0,055 \\
\hline Não elevado & & $121(49,4)$ & $16(34,8)$ & $9(42,9)$ & $96(53,9)$ & \\
\hline Elevado & & $124(50,6)$ & $30(65,2)$ & $12(57,1)$ & $82(46,1)$ & \\
\hline Localização, n(\%) & 205 & & & & & 0,094 \\
\hline $\begin{array}{l}\text { Não-cabeça e } \\
\text { Pescoço }\end{array}$ & & $136(66,3)$ & $25(65,8)$ & $16(88,9)$ & $95(63,8)$ & \\
\hline Cabeça e pescoço & & $69(33,7)$ & $13(34,2)$ & $2(11,1)$ & $54(36,2)$ & \\
\hline IMC, $n(\%)$ & 216 & & & & & 0,035 \\
\hline$<18$ & & $0(0,0)$ & $0(0,0)$ & $0(0,0)$ & $0(0,0)$ & \\
\hline
\end{tabular}




\begin{tabular}{|c|c|c|c|c|c|c|}
\hline$\geq 18, \leq 25$ & & $44(20,4)$ & $3(7,5)$ & $8(40,0)$ & $33(21,2)$ & \\
\hline$>25, \leq 30$ & & $78(36,1)$ & $20(50,0)$ & $5(25,0)$ & $53(34,0)$ & \\
\hline$>30$ & & $94(43,5)$ & $17(42,5)$ & $7(35,0)$ & $70(44,9)$ & \\
\hline BRAF, $n(\%)$ & 225 & & & & & 0,108 \\
\hline Sem mutação & & $153(68,0)$ & $24(58,5)$ & $15(75,0)$ & $114(69,5)$ & \\
\hline Outra mutação & & $17(7,6)$ & $1(2,4)$ & $0(0,0)$ & $16(9,8)$ & \\
\hline V600E & & $45(20,0)$ & $12(29,3)$ & $4(20,0)$ & $29(17,7)$ & \\
\hline V600K & & $10(4,4)$ & $4(9,8)$ & $1(5,0)$ & $5(3,0)$ & \\
\hline NRAS, n(\%) & 213 & & & & & 0,019 \\
\hline Sem mutação & & $167(78,4)$ & $35(85,4)$ & $10(52,6)$ & $122(79,7)$ & \\
\hline Mutação & & $46(21,6)$ & $6(14,6)$ & $9(47,4)$ & $31(20,3)$ & \\
\hline $\mathrm{KIT}, \mathrm{n}(\%)$ & 213 & & & & & 0,152 \\
\hline Sem mutação & & $186(87,3)$ & $33(86,8)$ & $14(73,7)$ & $139(89,1)$ & \\
\hline Mutação & & $27(12,7)$ & $5(13,2)$ & $5(26,3)$ & $17(10,9)$ & \\
\hline PTEN, $n(\%)$ & 46 & & & & & 1,000 \\
\hline Mutação & & $7(15,2)$ & $1(11,1)$ & $1(16,7)$ & $5(16,1)$ & \\
\hline Sem mutação & & $39(84,8)$ & $8(88,9)$ & $5(83,3)$ & $26(83,9)$ & \\
\hline Estágio, n(\%) & 245 & & & & & 0,042 \\
\hline Estágio III & & $22(9,0)$ & $1(2,2)$ & $0(0,0)$ & $21(11,8)$ & \\
\hline Estágio IV & & $223(91,0)$ & $45(97,8)$ & $21(100,0)$ & $157(88,2)$ & \\
\hline $\begin{array}{l}\text { Metástase linfonodal, } \\
\mathrm{n}(\%)\end{array}$ & 245 & & & & & 0,065 \\
\hline Não & & $65(26,5)$ & $6(13,0)$ & $6(28,6)$ & $53(29,8)$ & \\
\hline Sim & & $180(73,5)$ & $40(87,0)$ & $15(71,4)$ & $125(70,2)$ & \\
\hline $\begin{array}{l}\text { Metástase hepática, } \\
\mathrm{n}(\%)\end{array}$ & 245 & & & & & 0,523 \\
\hline Não & & $159(64,9)$ & $27(58,7)$ & $13(61,9)$ & $119(66,9)$ & \\
\hline Sim & & $86(35,1)$ & $19(41,3)$ & $8(38,1)$ & $59(33,1)$ & \\
\hline $\begin{array}{l}\text { Metástase pulmonar, } \\
\mathrm{n}(\%)\end{array}$ & 245 & & & & & 0,002 \\
\hline Não & & $99(40,4)$ & $9(19,6)$ & $7(33,3)$ & $83(46,6)$ & \\
\hline $\operatorname{Sim}$ & & $146(59,6)$ & $37(80,4)$ & $14(66,7)$ & $95(53,4)$ & \\
\hline $\begin{array}{l}\text { Metástase óssea, } \\
\mathrm{n}(\%)\end{array}$ & 245 & & & & & 0,302 \\
\hline Não & & $190(77,6)$ & $32(69,6)$ & $16(76,2)$ & $142(79,8)$ & \\
\hline Sim & & $55(22,4)$ & $14(30,4)$ & $5(23,8)$ & $36(20,2)$ & \\
\hline $\begin{array}{l}\text { Outras metástases } \\
\text { abdominais, } \mathrm{n}(\%)\end{array}$ & 245 & & & & & 0,438 \\
\hline Não & & $176(71,8)$ & $32(69,6)$ & $13(61,9)$ & $131(73,6)$ & \\
\hline $\operatorname{Sim}$ & & $69(28,2)$ & $14(30,4)$ & $8(38,1)$ & $47(26,4)$ & \\
\hline $\begin{array}{l}\text { Anti, BRAF prévio, } \\
\mathrm{n}(\%)\end{array}$ & 245 & & & & & 0,052 \\
\hline Não & & $195(79,6)$ & $31(67,4)$ & $16(76,2)$ & $148(83,1)$ & \\
\hline Sim & & $50(20,4)$ & $15(32,6)$ & $5(23,8)$ & $30(16,9)$ & \\
\hline $\begin{array}{l}\text { Ipilimumabe prévio, } \\
\mathrm{n}(\%)\end{array}$ & 245 & & & & & 0,229 \\
\hline Não & & $96(39,2)$ & $16(34,8)$ & $5(23,8)$ & $75(42,1)$ & \\
\hline Sim & & $149(60,8)$ & $30(65,2)$ & $16(76,2)$ & $103(57,9)$ & \\
\hline
\end{tabular}




\begin{tabular}{|c|c|c|c|c|c|c|}
\hline $\begin{array}{l}\text { Quimioterapia prévia } \\
\text { (Temozolamida), } \mathrm{n}(\%)\end{array}$ & 245 & & & & & $<0,001$ \\
\hline Não & & $197(80,4)$ & $32(69,6)$ & $10(47,6)$ & $155(87,1)$ & \\
\hline Sim & & $48(19,6)$ & $14(30,4)$ & $11(52,4)$ & $23(12,9)$ & \\
\hline $\begin{array}{l}\text { Quimioterapia prévia } \\
(\text { não-temozolamida*), } \\
\text { n(\%) }\end{array}$ & 245 & & & & & 0,009 \\
\hline Não & & $132(53,9)$ & $16(34,8)$ & $10(47,6)$ & $106(59,6)$ & \\
\hline Sim & & $113(46,1)$ & $30(65,2)$ & $11(52,4)$ & $72(40,4)$ & \\
\hline
\end{tabular}

Para toda a coorte de 320 pacientes, a mediana do tempo de acompanhamento foi de 31,2 (IIQ: 25,9 - 40,1) meses. A mediana e a média da duração do tratamento com anti-PD-1 foram 6,4 e 10,4 meses, respectivamente (amplitude: 0,5 - 62,6 e IIQ: 2,8 - 15,3 meses).

\subsection{Grupo "Sem Metástase Cerebral por Melanoma Prévia"}

Duzentos e quarenta e cinco pacientes (76,6\%) não tinham diagnostico de MCM antes do início do anti-PD-1 (Figura 1).

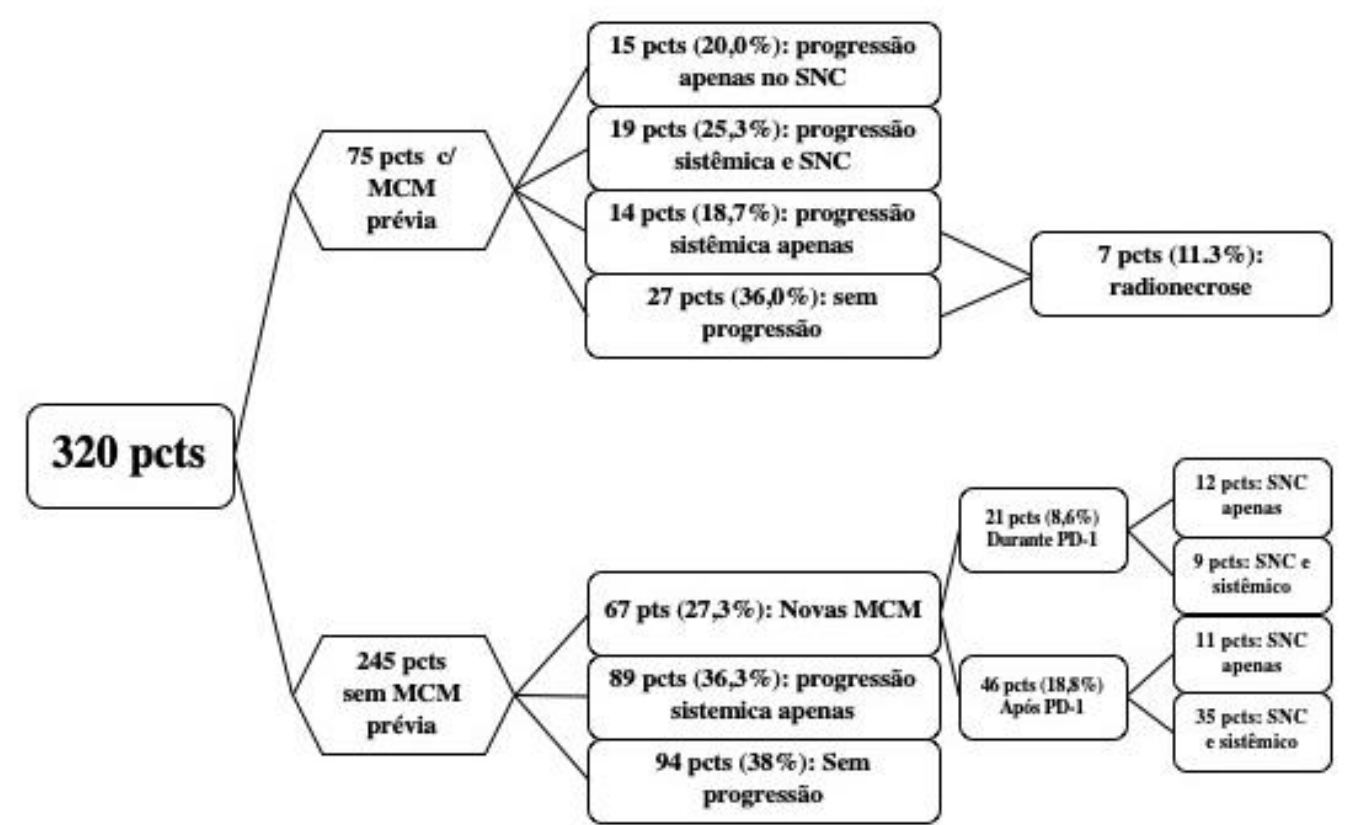

Pcts: pacientes; MCM: metástase cerebral por melanoma; SNC: sistema nervoso central; PD1: anti-PD-1.

Figura 1. Diagrama do estudo 
Até 0 momento de coleta dos dados, a mediana de acompanhamento desde o início do tratamento com anti-PD-1 foi de 33,5 (IIQ: 26,0 40,4) meses. A mediana do tempo de tratamento com terapia anti-PD-1 para este grupo foi de 6,0 meses (variando de 0,5 - 62,6 e IIQ: 2,8 - 14,3 meses) e a mediana de SG foi de 40,0 meses (intervalo de confiança de 95\% [IC 95\%]: 32,1 - 55,1 meses; Figura 2).

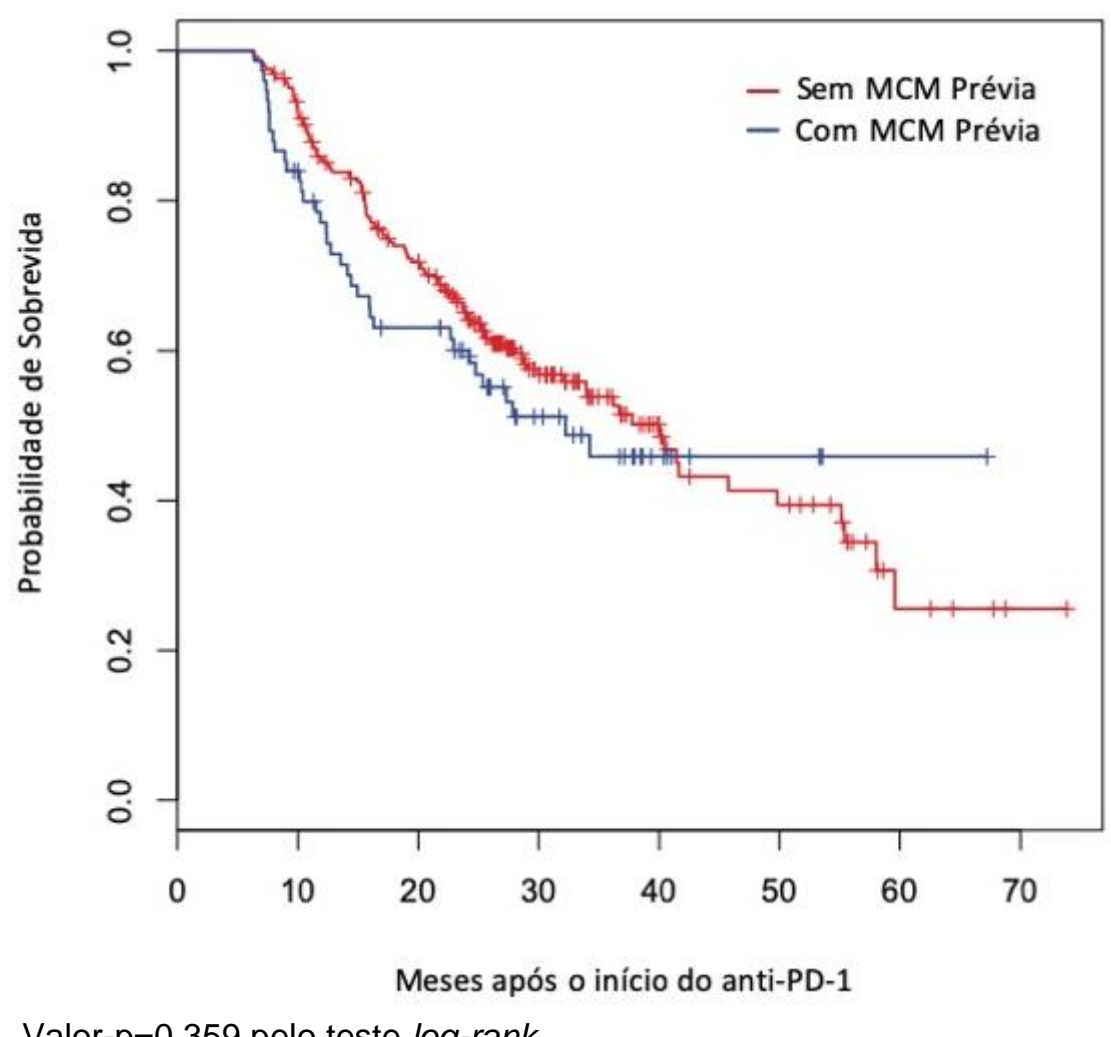

Valor- $\mathrm{p}=0,359$ pelo teste log-rank.

Figura 2. Sobrevida global de pacientes com e sem metástase cerebral prévia ao início de anti-PD-1

Um total de 67 pacientes sem doença intracraniana prévia desenvolveram MCM após o início do tratamento com anti-PD-1 (incidência de novas MCM: 27,3\%), incluindo 21 pacientes (8,6\%) durante a terapia anti-PD-1 e 46 pacientes $(18,8 \%)$ após a descontinuação do tratamento anti-PD-1. Entre os 21 pacientes que desenvolveram MCM durante o tratamento anti-PD-1, 12 foram diagnosticados com MCM sem evidência de progressão sistêmica, e 9 tiveram progressão concomitante no SNC e sistêmica. Entre os 46 pacientes que desenvolveram MCM 30 dias ou mais após o último tratamento com anti-PD-1, 11 tiveram apenas progressão do SNC, enquanto 35 progrediram em ambos (Figura 1). O número mediano de novas MCM foi 
de 3 para ambos os subgrupos. A mediana de SG para pacientes com progressão isolada em SNC foi, a partir do momento de diagnóstico da MCM, de 16,4 meses (IC 95\%: 7,3 - não atingido), enquanto para pacientes com progressão de sistêmica e em SNC, a SG mediana foi de 7,8 meses (95\% Cl: 6,2 10,2 meses). A SLMC mediana foi de 27,2 (95\% Cl: 22,1 - 37,7) meses (Figura 3).

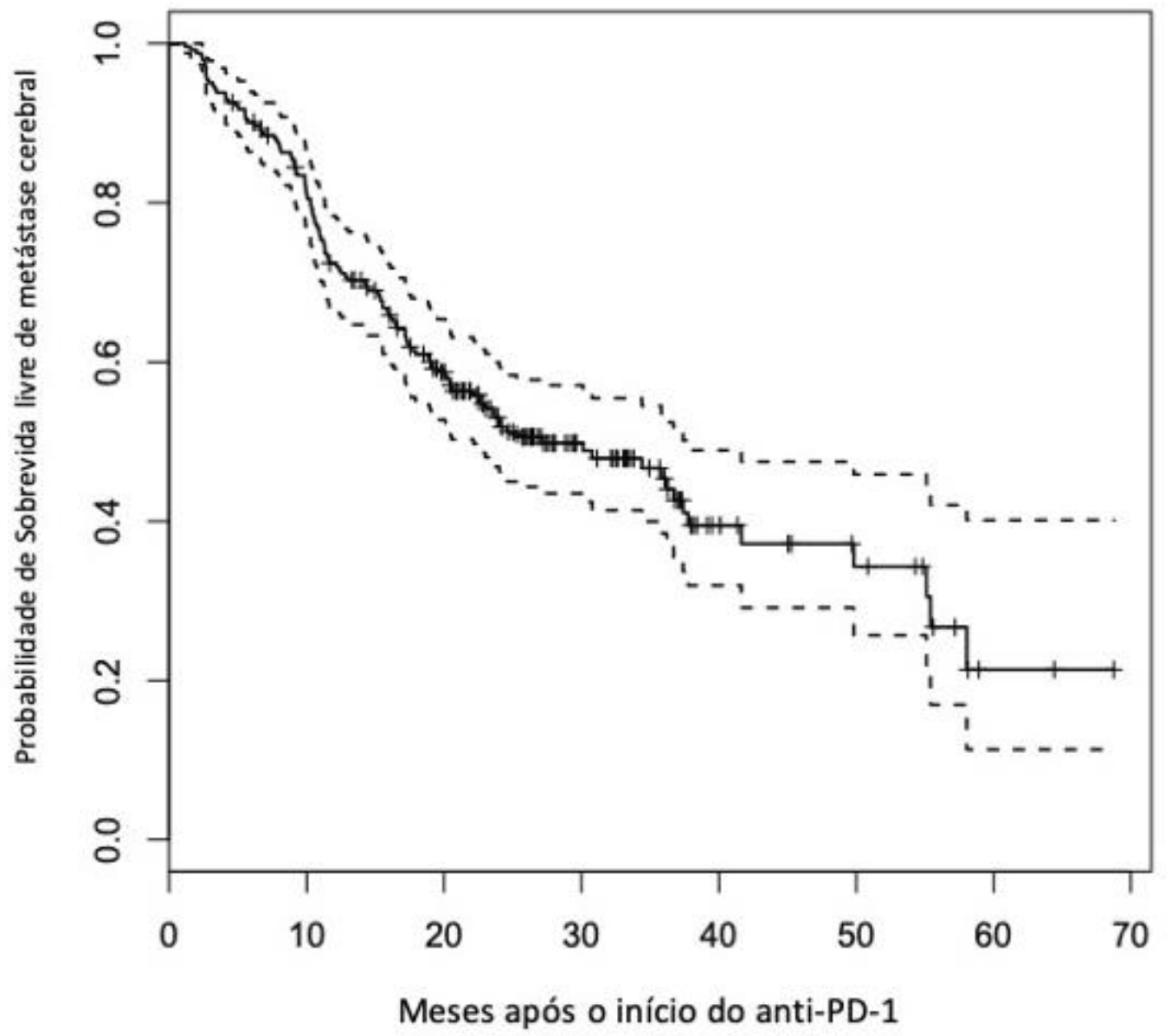

Figura 3. Sobrevida livre de metástase cerebral

$\mathrm{Na}$ análise multivariada, patologia primária de melanoma cutâneo acral ou uveal, bem como a exposição prévia à quimioterapia, foram associadas a pior SLMC (Tabela 3). 
Tabela 3. Análise uni/multivariada de fatores prognósticos para sobrevida livre de metástase cerebral

\begin{tabular}{|c|c|c|c|c|c|c|}
\hline \multirow{2}{*}{ Variável } & \multicolumn{3}{|c|}{ Univariada } & \multicolumn{3}{|c|}{ Multivariada } \\
\hline & HR & IC 95\% & Valor-p & HR & IC 95\% & Valor-p \\
\hline Idade & 0,99 & $(0,97,1,00)$ & 0,056 & 0,98 & $(0,97,1,00)$ & 0,024 \\
\hline \multicolumn{7}{|l|}{ Raça } \\
\hline Outra (ref) & - & - & - & & & \\
\hline Branco & 0,61 & $(0,34,1,11)$ & 0,104 & & & \\
\hline \multicolumn{7}{|l|}{ Patologia } \\
\hline Cutâneo, não-acral (ref) & - & - & - & - & - & - \\
\hline Cutâneo, acral & 2,79 & $(1,02,7,67)$ & 0,046 & 4,42 & $(1,53,12,80)$ & 0,006 \\
\hline Indeterminado & 0,77 & $(0,49,1,20)$ & 0,250 & 0,74 & $(0,46,1,16)$ & 0,189 \\
\hline Mucoso & 1,72 & $(0,95,3,11)$ & 0,074 & 1,76 & $(0,95,3,28)$ & 0,075 \\
\hline Uveal & 1,89 & $(0,97,3,68)$ & 0,060 & 2,38 & $(1,12,5,04)$ & 0,024 \\
\hline \multicolumn{7}{|l|}{ Ulceração } \\
\hline Ausente (ref) & - & - & - & & & \\
\hline Presente & 0,97 & $(0,62,1,52)$ & 0,889 & & & \\
\hline \multicolumn{7}{|l|}{$\mathrm{LDH}$} \\
\hline Não elevado (ref) & - & - & - & - & - & - \\
\hline Elevado & 1,48 & $(1,03,2,12)$ & 0,032 & 1,22 & $(0,83,1,78)$ & 0,317 \\
\hline \multicolumn{7}{|l|}{ Localização, n(\%) } \\
\hline $\begin{array}{l}\text { Não-cabeça e pescoço } \\
\text { (ref) }\end{array}$ & - & - & - & & & \\
\hline Cabeça e pescoço & 0,81 & $(0,54,1,21)$ & 0,294 & & & \\
\hline \multicolumn{7}{|l|}{ IMC, $n(\%)$} \\
\hline$\leq 30$ (ref) & - & - & - & & & \\
\hline$>30$ & 0,83 & $(0,57,1,21)$ & 0,323 & & & \\
\hline \multicolumn{7}{|l|}{ BRAF, n(\%) } \\
\hline Sem mutação (ref) & - & - & - & & & \\
\hline Outra mutação & 0,39 & $(0,14,1,07)$ & 0,067 & & & \\
\hline V600E & 0,91 & $(0,57,1,45)$ & 0,680 & & & \\
\hline V600K & 1,55 & $(0,67,3,56)$ & 0,303 & & & \\
\hline \multicolumn{7}{|l|}{ NRAS, $\mathrm{n}(\%)$} \\
\hline Sem mutação (ref) & - & - & - & & & \\
\hline Mutação & 1,45 & $(0,94,2,24)$ & 0,092 & & & \\
\hline \multicolumn{7}{|l|}{$\mathrm{KIT}, \mathrm{n}(\%)$} \\
\hline Sem mutação (ref) & - & - & - & & & \\
\hline Mutação & 1,45 & $(0,84,2,52)$ & 0,182 & & & \\
\hline \multicolumn{7}{|l|}{ PTEN, n(\%) } \\
\hline Mutação (ref) & - & - & - & & & \\
\hline Sem mutação & 1,30 & $(0,39,4,33)$ & 0,672 & & & \\
\hline \multicolumn{7}{|l|}{ Estágio, n(\%) } \\
\hline Estágio III & - & - & - & & & \\
\hline Estágio IV & 1,48 & $(0,75,2,91)$ & 0,262 & & & \\
\hline \multicolumn{7}{|l|}{$\begin{array}{l}\text { Metástase linfonodal, } \\
\mathrm{n}(\%)\end{array}$} \\
\hline Não (ref) & - & - & - & & & \\
\hline Sim & 1,09 & $(0,72,1,65)$ & 0,695 & & & \\
\hline \multicolumn{7}{|l|}{ Metástase hepática, n(\%) } \\
\hline Não (ref) & - & - & - & - & - & - \\
\hline
\end{tabular}




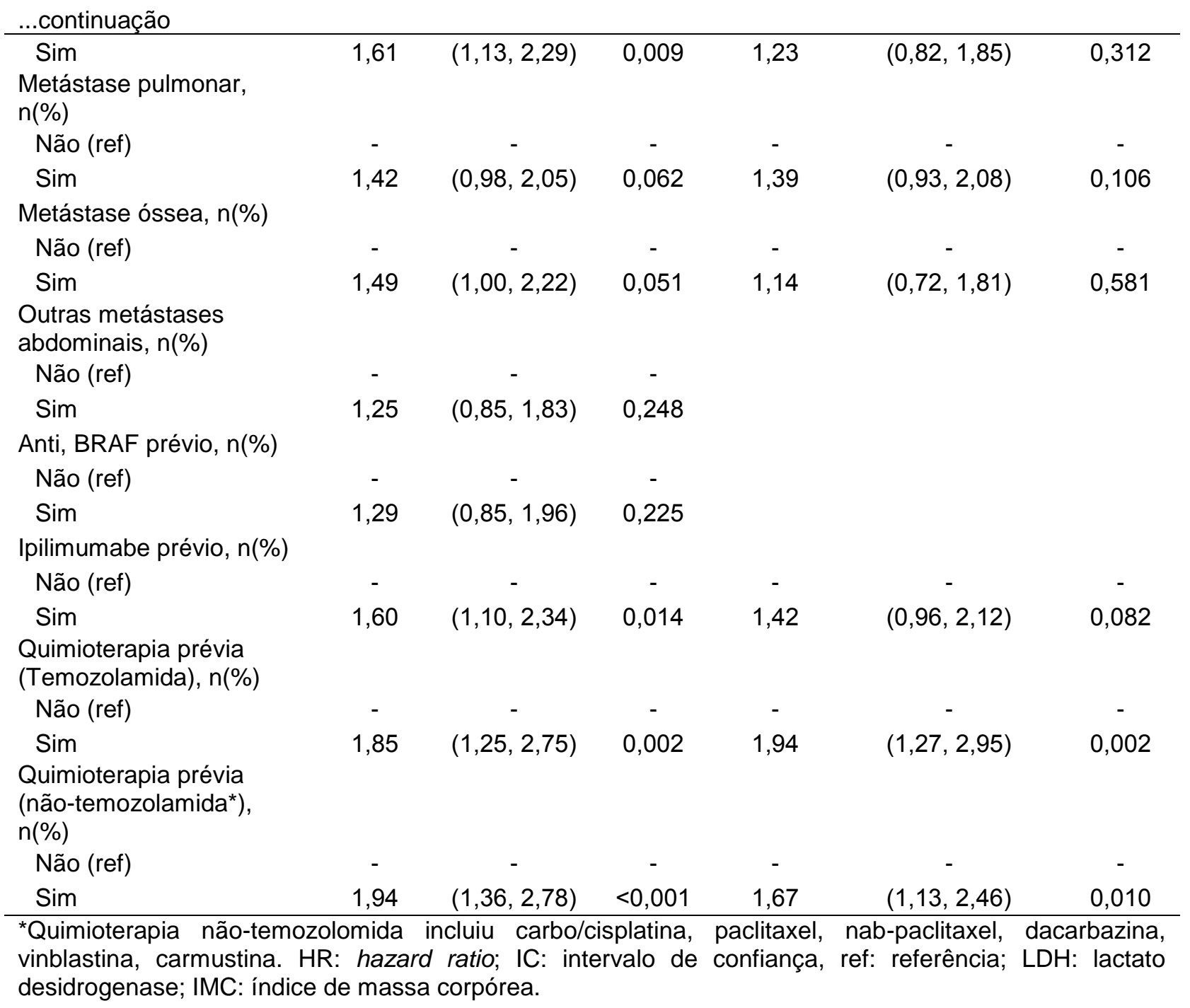

A SG mediana a partir do início do anti-PD-1 para pacientes que não desenvolveram MCM ( $\mathrm{N=178)}$ foi de 55,4 meses (IC 95\%: 49,8 - não atingido), que foi significativamente maior do que para pacientes que desenvolveram MCM durante ou após o anti-PD-1 ( $N=67$, mediana de 23,8 meses; IC 95\%: 20,6 - 32,1 meses; valor-p $<0,001)$. Entre os pacientes do grupo "Sem MCM Prévia", o desenvolvimento MCM durante ou após o anti-PD-1 foi associado com pior SG (HR: 4,70; IC 95\%: 3,18 - 6,93, $P<0,001)$, bem como a exposição prévia à quimioterapia (HR: 1,83; IC 95\%: 1,21 2,78; $P=0,004$ ) (Tabela 4). 
Tabela 4. Análise uni/multivariada de fatores prognósticos associados a sobrevida global em pacientes do grupo "Sem Metástase Cerebral por Melanoma Prévia"

\begin{tabular}{|c|c|c|c|c|c|c|}
\hline \multirow{2}{*}{ Variável } & \multicolumn{3}{|c|}{ Univariada } & \multicolumn{3}{|c|}{ Multivariada } \\
\hline & HR & IC 95\% & Valor-p & HR & IC 95\% & Valor-p \\
\hline Idade & 1.00 & $(0.98,1.01)$ & 0.682 & & & \\
\hline \multicolumn{7}{|l|}{ Raça } \\
\hline Outra (ref) & - & - & - & - & - & - \\
\hline Branco & 0.52 & $(0.28,0.98)$ & 0.042 & 0.66 & $(0.33,1.31)$ & 0.234 \\
\hline \multicolumn{7}{|l|}{ Patologia } \\
\hline Cutâneo, não-acral (ref) & - & - & - & - & - & - \\
\hline Cutâneo, acral & 4.09 & $(1.48,11.30)$ & 0.007 & 4.48 & $(1.42,14.13)$ & 0.010 \\
\hline Indeterminado & 0.77 & $(0.48,1.24)$ & 0.277 & 0.68 & $(0.42,1.13)$ & 0.135 \\
\hline Mucoso & 2.11 & $(1.15,3.86)$ & 0.015 & 1.36 & $(0.71,2.62)$ & 0.354 \\
\hline Uveal & 1.59 & $(0.76,3.35)$ & 0.219 & 1.74 & $(0.78,3.90)$ & 0.177 \\
\hline \multicolumn{7}{|l|}{ Ulceração } \\
\hline Ausente (ref) & - & - & - & & & \\
\hline Presente & 1.27 & $(0.79,2.02)$ & 0.322 & & & \\
\hline \multicolumn{7}{|l|}{$\mathrm{LDH}$} \\
\hline Não elevado (ref) & - & - & - & - & - & - \\
\hline Elevado & 1.28 & $(0.88,1.88)$ & 0.197 & & & \\
\hline \multicolumn{7}{|l|}{ Localização, n(\%) } \\
\hline $\begin{array}{l}\text { Não-cabeça e pescoço } \\
\text { (ref) }\end{array}$ & - & - & - & & & \\
\hline Cabeça e pescoço & 0.81 & $(0.53,1.24)$ & 0.334 & & & \\
\hline \multicolumn{7}{|l|}{$\mathrm{IMC}, \mathrm{n}(\%)$} \\
\hline$\leq 30$ (ref) & - & - & - & & & \\
\hline$>30$ & 0.90 & $(0.61,1.34)$ & 0.617 & & & \\
\hline \multicolumn{7}{|l|}{ BRAF, n(\%) } \\
\hline Sem mutação (ref) & - & - & - & & & \\
\hline Outra mutação & 0.35 & $(0.11,1.10)$ & 0.073 & & & \\
\hline V600E & 0.88 & $(0.54,1.45)$ & 0.626 & & & \\
\hline V600K & 0.62 & $(0.19,1.96)$ & 0.414 & & & \\
\hline \multicolumn{7}{|l|}{ NRAS, $n(\%)$} \\
\hline Sem mutação (ref) & - & - & - & & & \\
\hline Mutação & 1.53 & $(0.97,2.40)$ & 0.066 & & & \\
\hline \multicolumn{7}{|l|}{$\mathrm{KIT}, \mathrm{n}(\%)$} \\
\hline Sem mutação (ref) & - & - & - & & & \\
\hline Mutação & 1.27 & $(0.71,2.29)$ & 0.424 & & & \\
\hline \multicolumn{7}{|l|}{ PTEN, n(\%) } \\
\hline Mutação (ref) & - & - & - & & & \\
\hline Sem mutação & 4.69 & $(0.63,34.94)$ & 0.132 & & & \\
\hline \multicolumn{7}{|l|}{ Estágio, n(\%) } \\
\hline Estágio III & - & - & - & & & \\
\hline $\begin{array}{l}\text { Estágio IV } \\
\text { Metástase linfonodal, } \\
\mathrm{n}(\%)\end{array}$ & 1.13 & $(0.57,2.24)$ & 0.718 & & & \\
\hline Não (ref) & - & - & - & & & \\
\hline Sim & 1.08 & $(0.69,1.67)$ & 0.745 & & & \\
\hline
\end{tabular}


...continuação

Metástase hepática, $\mathrm{n}(\%)$

Não (ref)

Sim

1.87

$(1.29,2.73)$

0.001

1.54

$(0.98,2.43)$

0.061

Metástase pulmonar,

$\mathrm{n}(\%)$

Não (ref)

Sim

1.27

$(0.86,1.88)$

0.228

Metástase óssea, $\mathrm{n}(\%)$

Não (ref)

Sim

1.43

$(0.94,2.17)$

0.095

0.89

$(0.55,1.46)$

0.654

Outras metástases

abdominais, $n(\%)$

Não (ref)

Sim

1.28

$(0.85,1.91)$

0.238

Metástases cerebrais

Não (ref)

Sim

5.03

$(3.46,7.31)$

$<0.001$

4.70

$(3.18,6.93)$

$<0.001$

Número de MCM

1-3 (ref)

4-9

1.57

$(0.85,2.89)$

0.148

$10+$

1.22

$(0.50,2.99)$

0.660

Maior diâmetro de MCM

$<1 \mathrm{~cm}$ (ref)

$1-3 \mathrm{~cm}$

0.91

$(0.47,1.75)$

0.775

$>3 \mathrm{~cm}$

1.57

$(0.61,4.02)$

0.349

Anti, BRAF prévio, $n(\%)$

Não (ref)

Sim

1.10

$(0.70,1.73)$

0.684

Ipilimumabe prévio, $\mathrm{n}(\%)$

Não (ref)

Sim

1.76

$(1.17,2.65)$

0.007

1.57

$(1.01,2.43)$

0.045

Quimioterapia prévia

(Temozolamida), $\mathrm{n}(\%)$

Não (ref)

Sim

1.33

$(0.87,2.03)$

0.194

Quimioterapia prévia

(não-temozolamida*),

$\mathrm{n}(\%)$

Não (ref)

Sim

2.04

(1.39, 2.99)

$<0.001 \quad 1.83$

$(1.21,2.78) \quad 0.004$

${ }^{*}$ Quimioterapia não-temozolomida incluiu carbo/cisplatina, paclitaxel, nab-paclitaxel, dacarbazina, vinblastina, carmustina. HR: hazard ratio; IC: interval de confiança; ref: referência; LDH: lactato desidrogenase; IMC: índice de massa corpórea; MCM: metástase cerebral por melanoma.

Os resultados da análise multivariada para a coorte completa são exibidos na tabela 5 . 
Tabela 5. Análise uni/multivariada de fatores prognósticos associados a sobrevida global para todos os pacientes

\begin{tabular}{|c|c|c|c|c|c|c|}
\hline \multirow{2}{*}{ Variável } & \multicolumn{3}{|c|}{ Univariada } & \multicolumn{3}{|c|}{ Multivariada } \\
\hline & HR & IC 95\% & Valor-p & HR & IC 95\% & Valor-p \\
\hline Idade & 1,00 & $(0,99,1,01)$ & 0,967 & & & \\
\hline \multicolumn{7}{|l|}{ Raça } \\
\hline Outra (ref) & - & - & - & - & - & - \\
\hline Branco & 0,51 & $(0,30,0,89)$ & 0,018 & 0,74 & $(0,39,1,42)$ & 0,372 \\
\hline \multicolumn{7}{|l|}{ Patologia } \\
\hline Cutâneo, não-acral (ref) & - & - & - & - & - & - \\
\hline Cutâneo, acral & 3,79 & $(1,65,8,74)$ & 0,002 & 1,86 & $(0,69,5,00)$ & 0,220 \\
\hline Indeterminado & 1,11 & $(0,76,1,63)$ & 0,591 & 1,08 & $(0,73,1,61)$ & 0,691 \\
\hline Mucoso & 2,17 & $(1,24,3,79)$ & 0,006 & 1,81 & $(0,99,3,30)$ & 0,055 \\
\hline Uveal & 1,69 & $(0,84,3,38)$ & 0,139 & 2,49 & $(1,17,5,30)$ & 0,018 \\
\hline \multicolumn{7}{|l|}{ Ulceração } \\
\hline Ausente (ref) & - & - & - & & & \\
\hline Presente & 1,25 & $(0,82,1,91)$ & 0,297 & & & \\
\hline \multicolumn{7}{|l|}{ LDH } \\
\hline Não elevado (ref) & - & - & - & - & - & - \\
\hline Elevado & 1,60 & $(1,14,2,24)$ & 0,007 & 1,09 & $(0,74,1,61)$ & 0,657 \\
\hline \multicolumn{7}{|l|}{ Localização, n(\%) } \\
\hline $\begin{array}{l}\text { Não-cabeça e pescoço } \\
\text { (ref) }\end{array}$ & - & - & - & & & \\
\hline Cabeça e pescoço & 0,70 & $(0,47,1,05)$ & 0,082 & & & \\
\hline \multicolumn{7}{|l|}{$\mathrm{IMC}, \mathrm{n}(\%)$} \\
\hline$\leq 30$ (ref) & - & - & - & & & \\
\hline$>30$ & 1,05 & $(0,74,1,49)$ & 0,779 & & & \\
\hline \multicolumn{7}{|l|}{ BRAF, n(\%) } \\
\hline Sem mutação (ref) & - & - & - & & & \\
\hline Outra mutação & 0,60 & $(0,28,1,30)$ & 0,199 & & & \\
\hline V600E & 0,86 & $(0,57,1,29)$ & 0,467 & & & \\
\hline V600K & 0,71 & $(0,29,1,76)$ & 0,462 & & & \\
\hline \multicolumn{7}{|l|}{ NRAS, n(\%) } \\
\hline Sem mutação (ref) & - & - & - & & & \\
\hline Mutação & 1,38 & $(0,94,2,04)$ & 0,101 & & & \\
\hline \multicolumn{7}{|l|}{$\mathrm{KIT}, \mathrm{n}(\%)$} \\
\hline Sem mutação (ref) & - & - & - & & & \\
\hline Mutação & 1,48 & $(0,92,2,40)$ & 0,109 & & & \\
\hline \multicolumn{7}{|l|}{ PTEN, n(\%) } \\
\hline Mutação (ref) & - & - & - & & & \\
\hline Sem mutação & 3,58 & $(0,85,15,00)$ & 0,081 & & & \\
\hline \multicolumn{7}{|l|}{ Estágio, n(\%) } \\
\hline Estágio III (ref) & - & - & - & & & \\
\hline Estágio IV & 1,17 & $(0,60,2,31)$ & 0,641 & & & \\
\hline \multicolumn{7}{|l|}{ Metástase linfonodal, n(\%) } \\
\hline Não (ref) & - & - & - & & & \\
\hline $\operatorname{Sim}$ & 0,88 & $(0,61,1,27)$ & 0,496 & & & \\
\hline \multicolumn{7}{|l|}{ Metástase hepática, n(\%) } \\
\hline Não (ref) & - & - & - & - & - & - \\
\hline
\end{tabular}




\begin{tabular}{|c|c|c|c|c|c|c|}
\hline Sim & 1,61 & $(1,17,2,23)$ & 0,004 & 0,97 & $(0,66,1,41)$ & 0,860 \\
\hline \multicolumn{7}{|c|}{ Metástase pulmonar, n(\%) } \\
\hline Não (ref) & - & - & - & & & \\
\hline Sim & 1,05 & $(0,75,1,47)$ & 0,788 & & & \\
\hline \multicolumn{7}{|c|}{ Metástase óssea, n(\%) } \\
\hline Não (ref) & - & - & - & & & \\
\hline Sim & 1,31 & $(0,91,1,89)$ & 0,152 & & & \\
\hline \multicolumn{7}{|c|}{$\begin{array}{l}\text { Outras metástases } \\
\text { abdominais, } \mathrm{n}(\%)\end{array}$} \\
\hline Não (ref) & - & - & - & - & - & - \\
\hline Sim & 1,34 & $(0,96,1,88)$ & 0,084 & 1,06 & $(0,72,1,54)$ & 0,771 \\
\hline \multicolumn{7}{|c|}{ Metástases cerebrais } \\
\hline Não (ref) & - & - & - & - & - & - \\
\hline Sim & 3,07 & $(2,21,4,27)$ & $<0,001$ & 3,25 & $(2,26,4,68)$ & $<0,001$ \\
\hline \multicolumn{7}{|c|}{$\begin{array}{l}\text { Número de metástases } \\
\text { cerebrais }\end{array}$} \\
\hline $4-9$ & 1,50 & $(0,93,2,42)$ & 0,099 & & & \\
\hline $10+$ & 1,82 & $(1,04,3,19)$ & 0,037 & & & \\
\hline \multicolumn{7}{|c|}{$\begin{array}{l}\text { Maior diâmetro de } \\
\text { metástases cerebrais }\end{array}$} \\
\hline$<1 \mathrm{~cm}$ (ref) & - & - & - & & & \\
\hline $1-3 \mathrm{~cm}$ & 1,09 & $(0,63,1,90)$ & 0,758 & & & \\
\hline$>3 \mathrm{~cm}$ & 1,93 & $(1,00,3,70)$ & 0,050 & & & \\
\hline \multicolumn{7}{|c|}{ Anti, BRAF prévio, $\mathrm{n}(\%)$} \\
\hline Não (ref) & - & - & - & & & \\
\hline $\operatorname{Sim}$ & 1,13 & $(0,78,1,64)$ & 0,506 & & & \\
\hline \multicolumn{7}{|c|}{ Ipilimumabe prévio, $\mathrm{n}(\%)$} \\
\hline Não (ref) & - & - & - & - & - & - \\
\hline Sim & 1,80 & $(1,26,2,58)$ & 0,001 & 1,69 & $(1,15,2,47)$ & 0,007 \\
\hline \multicolumn{7}{|c|}{$\begin{array}{l}\text { Quimioterapia prévia } \\
\text { (Temozolamida), n(\%) }\end{array}$} \\
\hline Não (ref) & - & - & - & - & - & - \\
\hline Sim & 1,57 & $(1,10,2,23)$ & 0,012 & 1,08 & $(0,73,1,60)$ & 0,699 \\
\hline \multicolumn{7}{|c|}{$\begin{array}{l}\text { Quimioterapia prévia (não- } \\
\text { temozolamida*), } \mathrm{n}(\%)\end{array}$} \\
\hline Não (ref) & - & - & - & - & - & - \\
\hline Sim & 2,13 & $(1,54,2,96)$ & $<0,001$ & 2,12 & $(1,48,3,05)$ & $<0,001$ \\
\hline
\end{tabular}

${ }^{*}$ Quimioterapia não-temozolomida incluiu carbo/cisplatina, paclitaxel, nab-paclitaxel, dacarbazina, vinblastina, carmustina. HR: hazard ratio; IC: interval de confiança; ref: referência; LDH: lactato desidrogenase; IMC: índice de massa corpórea.

O tempo mediano de SG a partir da data do diagnóstico de MCM até a última data vital, no grupo "Sem MCM Prévia" ( $N=67$ ), foi de 8,7 meses (IC 95\%: 6,912,3). Da mesma forma, ao analisar por subgrupos "Durante Anti-PD-1" ( $\mathrm{N}=21)$ e "Após Ant-PD-1" ( $N=46)$, a mediana da SG foi de 10,4 (IC 95\%: 6,9 - não atingido) e 8,0 meses (IC 95\%: 6,2 - 12,2 meses), respectivamente (Figura 4). 


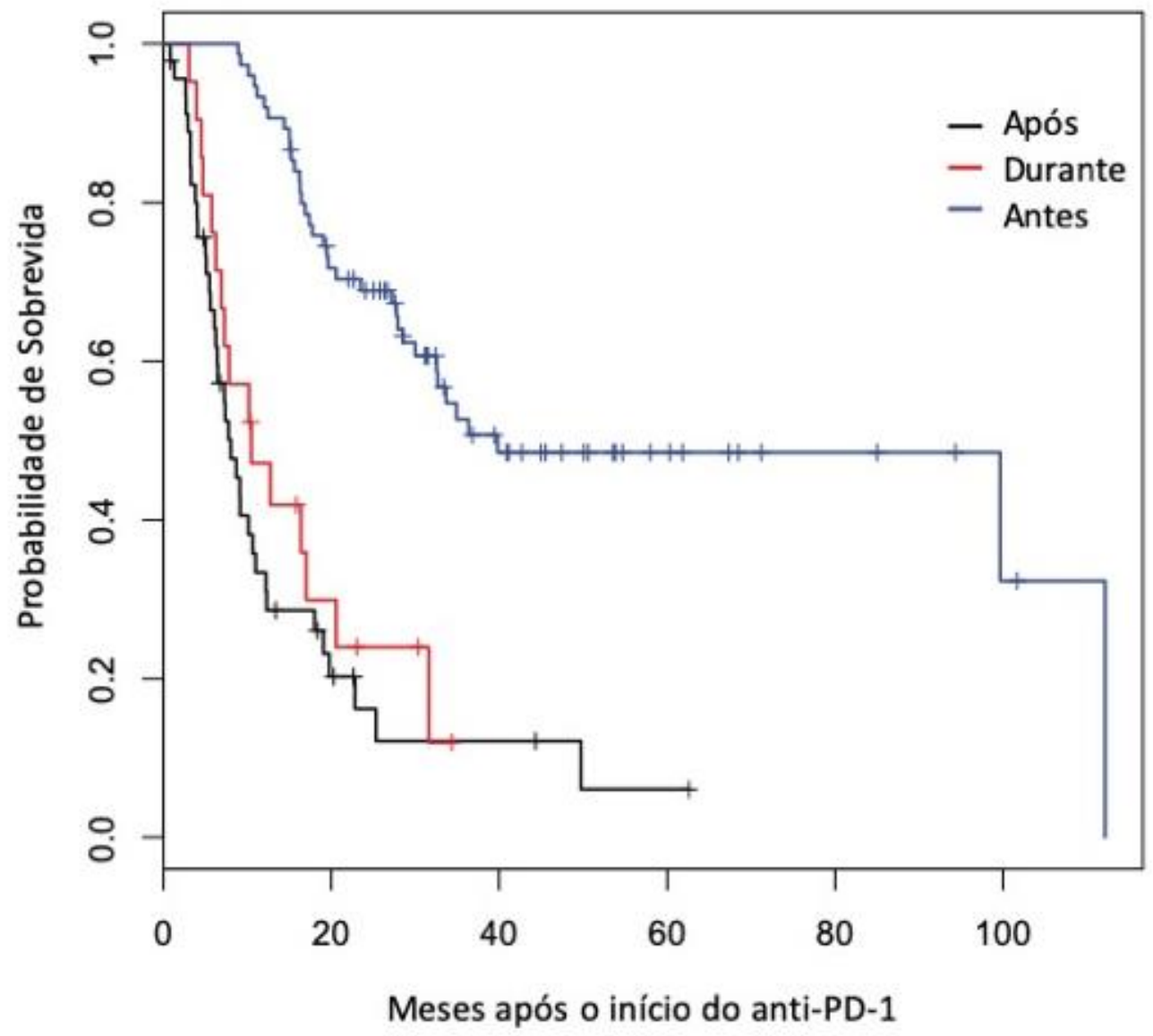

Valor-p $<0,001$ pelo teste log-rank.

Figura 4. Sobrevida global de pacientes de acordo com o momento de diagnóstico de metástases cerebrais

No grupo "Com MCM Prévia", a SG mediana correspondente foi de 39,9 meses (IC 95\%: 32,5 - não alcançada) a partir da data do diagnóstico de MCM.

\subsection{Grupo "Com Metástase Cerebral por Melanoma Prévia"}

Setenta e cinco pacientes $(23,4 \%)$ foram diagnosticados com MCM antes do tratamento com anti-PD-1. Todos, com exceção de 4 pacientes, receberam terapia local dirigida ao SNC (radioterapia ou cirurgia) antes do primeiro tratamento anti-PD-1. A duração mediana do tratamento com terapia anti-PD-1 foi de 8,8 meses (amplitude: 0,7 - 33,1 e IIQ: 3,0 - 19,6 meses), e a SG mediana foi de 32,2 (IC 95\%: 22,9 - não atingida) meses. O tempo mediano desde o diagnóstico inicial de MCM até 0 início do anti-PD-1 foi de 5,4 (IIQ: 1,7 - 13,1) meses e o tempo médio de acompanhamento do início do anti-PD-1 foi de 33,5 (IIQ: 26,0 - 40,4) meses. A SG desde 0 início do tratamento anti-PD-1 (mediana: 32,2 meses) não foi 
significativamente diferente em comparação com os pacientes no grupo "Sem MCM Prévia" ( $P=0,359$, Figura 2).

Um total de 34 (45,3\%) dos pacientes apresentou progressão da doença no SNC, incluindo 15 (20,0\%) com progressão apenas no SNC e 19 (25,3\%) com progressão sistêmica e no SNC. Treze pacientes $(17,3 \%)$ progrediram apenas sistemicamente e 27 (36,0\%) não haviam progredido até a coleta dos dados (Figura 1). A SG mediana para pacientes que apresentaram apenas progressão do SNC nesse grupo foi de 39,9 meses (IC 95\%: 30,0 - não alcançada), comparada a 17,8 meses (IC 95\%: 15,3 - 32,5 meses) para pacientes que desenvolveram progressão sistêmica e em SNC.

A SNC-SLP mediana foi de 10,4 meses (IC 95\%: 7,8 - 24,3 meses), e a mediana de SIS-SLP foi de 16,0 meses (IC 95\%: 9,6 - não atingido). Pacientes com LDH elevado apresentaram pior SNC-SLP (HR: 1,95; IC 95\%: 1,03 - 3,70; P = 0,040) e pior SIS-SLP (HR: 3,13; IC 95\%: 1,49 - 6,58; $\mathrm{P}=0,003$ ), assim como pacientes que receberam quimioterapia prévia (Tabela 6).

Tabela 6. Análise univariada de fatores prognósticos associados com SNC-SLP e SIS-SLP entre pacientes do grupo "Com Metástase Cerebral por Melanoma Prévia"

\begin{tabular}{|c|c|c|c|c|c|c|}
\hline \multirow{2}{*}{ Variável } & \multicolumn{3}{|c|}{ SNC-SLP } & \multicolumn{3}{|c|}{ SIS-SLP } \\
\hline & HR & IC 95\% & Valor-p & HR & IC 95\% & Valor-p \\
\hline Idade & 1,01 & $(0,99,1,03)$ & 0,381 & 1,00 & $(0,98,1,02)$ & 0,865 \\
\hline \multicolumn{7}{|l|}{ Raça } \\
\hline Outra (ref) & - & - & - & - & - & - \\
\hline Branco & 0,58 & $(0,21,1,63)$ & 0,303 & 0,61 & $(0,19,1,99)$ & 0,414 \\
\hline \multicolumn{7}{|l|}{ Patologia } \\
\hline Cutâneo, não-acral (ref) & - & - & - & - & - & - \\
\hline Cutâneo, acral & 2,91 & $(0,68,12,43)$ & 0,150 & 2,99 & $(0,69,12,88)$ & 0,142 \\
\hline Indeterminado & 1,96 & $(1,07,3,61)$ & 0,030 & 1,66 & $(0,85,3,25)$ & 0,137 \\
\hline Mucoso & 2,31 & $(0,69,7,78)$ & 0,176 & 4,01 & $(1,18,13,63)$ & 0,026 \\
\hline Uveal & 1,49 & $(0,20,11,10)$ & 0,696 & 2,23 & $(0,30,16,73)$ & 0,436 \\
\hline \multicolumn{7}{|l|}{ Ulceração } \\
\hline Ausente (ref) & - & - & - & - & - & - \\
\hline Presente & 1,28 & $(0,55,2,96)$ & 0,567 & 1,48 & $(0,60,3,66)$ & 0,400 \\
\hline \multicolumn{7}{|l|}{ LDH } \\
\hline Não elevado (ref) & - & - & - & - & - & - \\
\hline Elevado & 1,95 & $(1,03,3,70)$ & 0,040 & 3,13 & $(1,49,6,58)$ & 0,003 \\
\hline \multicolumn{7}{|l|}{ Localização, n(\%) } \\
\hline $\begin{array}{l}\text { Não-cabeça e pescoço } \\
\text { (ref) }\end{array}$ & - & - & - & - & - & - \\
\hline Cabeça e pescoço & 0,56 & $(0,23,1,38)$ & 0,209 & 0,40 & $(0,14,1,15)$ & 0,090 \\
\hline IMC, n(\%) & & & & & & \\
\hline
\end{tabular}




\begin{tabular}{|c|c|c|c|c|c|c|}
\hline$\leq 30$ (ref) & - & - & - & - & - & - \\
\hline$>30$ & 1,03 & $(0,53,1,99)$ & 0,934 & 1,30 & $(0,66,2,56)$ & 0,454 \\
\hline \multicolumn{7}{|l|}{ BRAF, n(\%) } \\
\hline Sem mutação (ref) & - & - & - & - & - & - \\
\hline Outra mutação & 0,91 & $(0,28,3,02)$ & 0,883 & 1,12 & $(0,39,3,28)$ & 0,829 \\
\hline V600E & 0,63 & $(0,33,1,21)$ & 0,168 & 0,98 & $(0,49,1,96)$ & 0,958 \\
\hline V600K & 1,32 & $(0,46,3,81)$ & 0,602 & 0,84 & $(0,20,3,59)$ & 0,815 \\
\hline \multicolumn{7}{|l|}{ NRAS, n(\%) } \\
\hline Sem mutação (ref) & - & - & - & - & - & - \\
\hline Mutação & 1,55 & $(0,81,2,98)$ & 0,186 & 1,17 & $(0,58,2,35)$ & 0,670 \\
\hline \multicolumn{7}{|l|}{$\mathrm{KIT}, \mathrm{n}(\%)$} \\
\hline Sem mutação (ref) & - & - & - & - & - & - \\
\hline Mutação & 1,63 & $(0,71,3,70)$ & 0,246 & 1,44 & $(0,63,3,28)$ & 0,392 \\
\hline \multicolumn{7}{|l|}{ PTEN, $n(\%)$} \\
\hline Mutação (ref) & - & - & - & - & - & - \\
\hline Sem mutação & 0,93 & $(0,20,4,22)$ & 0,925 & 1,85 & $(0,24,14,49)$ & 0,558 \\
\hline \multicolumn{7}{|c|}{ Metástase linfonodal, $n(\%)$} \\
\hline Não (ref) & - & - & - & - & - & - \\
\hline Sim & 0,69 & $(0,38,1,26)$ & 0,228 & 0,74 & $(0,37,1,46)$ & 0,381 \\
\hline \multicolumn{7}{|c|}{ Metástase hepática, n(\%) } \\
\hline Não (ref) & - & - & - & - & - & - \\
\hline Sim & 1,00 & $(0,57,1,77)$ & 0,994 & 1,22 & $(0,66,2,26)$ & 0,530 \\
\hline \multicolumn{7}{|c|}{ Metástase pulmonar n(\%) } \\
\hline Não (ref) & - & - & - & - & - & - \\
\hline $\operatorname{Sim}$ & 0,86 & $(0,46,1,58)$ & 0,619 & 0,58 & $(0,31,1,10)$ & 0,097 \\
\hline \multicolumn{7}{|l|}{ Metástase óssea, n(\%) } \\
\hline Não (ref) & - & - & - & - & - & - \\
\hline $\operatorname{Sim}$ & 0,89 & $(0,44,1,78)$ & 0,739 & 1,16 & $(0,57,2,36)$ & 0,689 \\
\hline \multicolumn{7}{|l|}{$\begin{array}{l}\text { Outras metástases } \\
\text { abdominais, } \mathrm{n}(\%)\end{array}$} \\
\hline Não (ref) & - & - & - & - & - & - \\
\hline $\operatorname{Sim}$ & 0,91 & $(0,52,1,62)$ & 0,759 & 1,70 & $(0,91,3,17)$ & 0,095 \\
\hline \multicolumn{7}{|c|}{ Anti, BRAF prévio, $\mathrm{n}(\%)$} \\
\hline Não (ref) & - & - & - & - & - & - \\
\hline $\operatorname{Sim}$ & 0,88 & $(0,48,1,60)$ & 0,669 & 1,28 & $(0,67,2,43)$ & 0,454 \\
\hline \multicolumn{7}{|c|}{ Ipilimumabe prévio, $\mathrm{n}(\%)$} \\
\hline Não (ref) & - & - & - & - & - & - \\
\hline $\operatorname{Sim}$ & 1,60 & $(0,88,2,93)$ & 0,123 & 1,68 & $(0,86,3,30)$ & 0,130 \\
\hline \multicolumn{7}{|l|}{$\begin{array}{l}\text { Quimioterapia prévia } \\
\text { (Temozolamida), n(\%) }\end{array}$} \\
\hline Não (ref) & - & - & - & - & - & - \\
\hline Sim & 2,20 & $(1,24,3,91)$ & 0,007 & 2,50 & $(1,35,4,63)$ & 0,003 \\
\hline \multicolumn{7}{|c|}{$\begin{array}{l}\text { Quimioterapia prévia (não- } \\
\text { temozolamida*), } n(\%)\end{array}$} \\
\hline Não (ref) & - & - & - & - & - & - \\
\hline $\operatorname{Sim}$ & 2,05 & $(1,16,3,63)$ & 0,013 & 2,24 & $(1,20,4,17)$ & 0,011 \\
\hline Radiocirurgia estereo & & & & & & \\
\hline
\end{tabular}




\begin{tabular}{|c|c|c|c|c|c|c|}
\hline Não (ref) & - & - & - & - & - & - \\
\hline $\operatorname{Sim}$ & 1,64 & $(0,64,4,19)$ & 0,301 & 2,40 & $(1,02,5,67)$ & 0,046 \\
\hline \multicolumn{7}{|c|}{$\begin{array}{l}\text { Radioterapia cranial } \\
\text { total }\end{array}$} \\
\hline Não (ref) & - & - & - & - & - & - \\
\hline $\operatorname{Sim}$ & 2,01 & $(1,08,3,74)$ & 0,027 & 1,44 & $(0,82,2,55)$ & 0,208 \\
\hline \multicolumn{7}{|c|}{ Craniotomia } \\
\hline Não (ref) & - & - & - & - & - & - \\
\hline Sim & 0,82 & $(0,43,1,55)$ & 0,547 & 0,76 & $(0,42,1,38)$ & 0,372 \\
\hline
\end{tabular}

\subsection{Radionecrose durante o tratamento com anti-PD-1}

Sete pacientes do grupo "Com MCM Prévia" desenvolveram radionecrose $(\mathrm{RN})$. Todos os pacientes haviam recebido radiocirurgia estereotáxica (stereotactic radiosurgery, SRS) prévia à lesões pré-existentes no SNC antes de iniciar o tratamento com anti-PD1 (11,3\% dos 62 pacientes que receberam SRS antes do início do tratamento). O tempo mediano para o desenvolvimento de $\mathrm{RN}$ a partir da data de início do anti-PD-1 foi de 5,5 (IIQ: 4,3 - 16,6) meses e o tempo mediano para RN da última radiação foi de 7,5 meses (IIQ: 4,5 - 17,4). A interrupção do agente anti-PD1 foi suficiente para diminuir o edema em um paciente, mas os outros seis pacientes receberam tratamento com dexametasona. Dois necessitaram de craniotomia e um paciente foi tratado com Terapia Intersticial por Laser (Laser Interstitial Thermal Therapy, LITT). No momento do diagnóstico de RN, nenhum dos pacientes apresentou progressão da doença sistêmica concomitante.

\subsection{Tratamento de metástase cerebral por melanoma}

A terapia dirigida para as MCM que ocorreram após o início do antiPD-1 incluiu SRS em 41 pacientes $(61,2 \%)$ e radiação cerebral total em 14 pacientes (20,9\%, Tabela 7$)$. 
Tabela 7. Resumo do tratamento local direcionado ao cérebro

\begin{tabular}{lcccc}
\hline Variável & $\mathbf{n}$ & Total & $\begin{array}{c}\text { Sem MCM } \\
\text { prévia }\end{array}$ & $\begin{array}{c}\text { Com MCM } \\
\text { prévia }\end{array}$ \\
\hline $\begin{array}{l}\text { Alguma modalidade de } \\
\text { tratamento, } \mathrm{n}(\%)\end{array}$ & 142 & & & \\
$\quad$ Não & & $20(14,1)$ & $16(23,9)$ & $4(5,3)$ \\
$\quad$ Sim & & $122(85,9)$ & $51(76,1)$ & $71(94,7)$ \\
Radiocirurgia estereotáxica, n(\%) & 142 & & & \\
$\quad$ Não & & $39(27,5)$ & $26(38,8)$ & $13(17,3)$ \\
$\quad$ Sim & & $103(72,5)$ & $41(61,2)$ & $62(82,7)$ \\
Radioterapia cranial total, n(\%) & 142 & & & \\
$\quad$ Não & & $94(66,2)$ & $53(79,1)$ & $41(54,7)$ \\
$\quad$ Sim & 142 & $48(33,8)$ & $14(20,9)$ & $34(45,3)$ \\
Craniotomia, n(\%) & & & \\
$\quad$ Não & & $101(71,1)$ & $54(80,6)$ & $47(62,7)$ \\
$\quad$ Sim & & $41(28,9)$ & $13(19,4)$ & $28(37,3)$ \\
Duas ou mais modalidades, $\mathrm{n}(\%)$ & 142 & & & \\
$\quad$ Não & & $85(59,9)$ & $53(79,1)$ & $32(42,7)$ \\
$\quad$ Sim & & $57(40,1)$ & $14(20,9)$ & $43(57,3)$ \\
\hline
\end{tabular}

MCM: metástase cerebral por melanoma.

Treze pacientes $(19,4 \%)$ também necessitaram de craniotomia. Cinquenta e um pacientes $(76,1 \%)$ tiveram pelo menos uma modalidade de tratamento, enquanto $14(20,9 \%)$ tiveram dois ou mais.

Em pacientes com MCM prévia, a SRS foi usada em 62 pacientes (82,7\%), e a radiação cerebral total foi escolhida em 34 pacientes $(45,3 \%)$. Um total de 28 pacientes $(37,3 \%)$ necessitaram de craniotomia como parte do manejo da MCM, com 43 pacientes $(57,3 \%)$ necessitando de pelo menos duas modalidades de tratamento diferentes. Apenas $4(5,3 \%)$ dos pacientes deste grupo não receberam terapia local antes do início do anti-PD-1. 


\section{DISCUSSÃO}

Embora o panorama do tratamento do melanoma tenha mudado dramaticamente nos últimos sete anos, várias questões permanecem em aberto, incluindo como esses novos agentes podem afetar o desenvolvimento de novas MCM e impedir a progressão das lesões pré-existentes. O presente estudo é o maior relato retrospectivo dos resultados de desfechos e padrões de progressão no cérebro em pacientes com melanoma tratados com anti-PD-1, e o primeiro a relatar a incidência de novas MCM durante ou após a exposição ao imunoterápico. Observamos uma incidência de MCM de 8,6\% durante o tratamento anti-PD-1 e de 18,8\% após o tratamento com anti-PD-1 em pacientes sem evidência de doença no SNC antes do início da terapia com anti-PD-1. Ademais, descobrimos que a progressão isolada da doença intracraniana ocorreu frequentemente, na ausência de progressão sistêmica concomitante.

Historicamente, a presença de MCM está associada à pior sobrevida e considerada um fator prognóstico ruim. ${ }^{(16)}$ No entanto, dados sobre como os agentes anti-neoplásicos recentemente aprovados para o tratamento de melanoma podem afetar o desenvolvimento de MCM são escassos. Com terapia alvo-dirigida, alguns estudos foram publicados sobre a incidência e padrões de progressão de MCM. Gummadi et al. demonstraram que pacientes com melanoma que apresentam mutação no gene BRAF e receberam vemurafenibe tiveram diminuição da incidência de metástases cerebrais em comparação com aqueles que não o receberam. ${ }^{(32)}$ Peuvrel et al. encontraram uma incidência de $20 \%$ de novas MCM em pacientes tratados com vemurafenibe, dos quais 59\% tinham doença sistêmica controlada no momento da progressão do SNC. ${ }^{(33)}$ No entanto, não encontramos estudos avaliando as mesmas questões durante a terapia com anti-PD-1.

Em nossa coorte, não observamos menor sobrevida a partir do início de anti-PD-1 em pacientes com MCM previamente diagnosticada, em comparação com aqueles sem doença intracraniana, mesmo com 45,3\% dos pacientes no grupo "MCM Prévia" apresentando progressão em SNC, comparado com 27,3\% de incidência de novas MCM durante ou após o uso de anti-PD-1 em pacientes sem MCM prévia conhecida. A SG mediana do momento do diagnóstico de MCM em pacientes com MCM prévia foi muito superior ao controle histórico (39,9 meses). Isso pode indicar que 
a terapia anti-PD-1 pode atenuar a natureza agressiva conhecida por essa população, embora também possa ser explicada por um viés de seleção de pacientes que sobreviveram tempo suficiente para tolerar linhas sucessivas de terapia. Não obstante, a coorte sem MCM prévia teve uma incidência menor de novas MCM em comparação ao que seria esperado para essa população. Embora o seguimento mediano de 31,2 meses tenha sido relativamente curto para tal asserção, os pacientes que desenvolveram MCM foram tipicamente diagnosticados no primeiro ano após o início do anti-PD-1 (o tempo mediano para diagnóstico de novas MCM em pacientes sem MCM prévia foi de 9,9 meses). Ainda assim, o desenvolvimento de MCM durante o tratamento com anti-PD-1 foi associado de forma independente com pior sobrevida na análise multivariada. Além disso, quando analisado como uma variável covariante no tempo e, portanto, abrangendo o diagnóstico de MCM ao longo de todo o curso da doença até o momento, a MCM foi um forte preditor de pior SG. O tempo mediano de SG calculado a partir da data do diagnóstico MCM até a última data vital no grupo "Sem MCM Prévia" foi consideravelmente menor do que no grupo "Com MCM Prévia" (8.7 versus 39.9 meses, respectivamente), implicando que ter um novo diagnóstico de metástase cerebral após exposição ao anti-PD-1 representa um desafio terapêutico e confere um mau prognóstico no cenário de resistência inata ou adquirida à imunoterapia (Figura 4).

No entanto, a mediana de sobrevida para pacientes com MCM foi significativamente maior do que o controle histórico da era pré-anti-PD-1. Trinta e seis por cento dos pacientes com MCM prévia não haviam progredido até o momento de fechamento dos dados (mediana de acompanhamento para o grupo: 33,5 meses), uma porcentagem que é comparável com os dados de longo prazo dos estudos de monoterapia anti-PD-1. ${ }^{(34)}$ Além disso, dados recentes revelaram que a adição de ipilimumabe ao anti-PD-1 pode dobrar as taxas de resposta intracraniana e prolongar a PFS intracraniana, com controle duradouro do SNC e fornecendo novas evidências de que essa população pode compartilhar os mesmos benefícios de longo prazo da imunoterapia que pacientes sem MCM ativa apresentam. ${ }^{(28,29)}$

Curiosamente, cerca de metade dos pacientes em nosso estudo apresentaram doença extracraniana controlada no momento da progressão no SNC. Isso corrobora a teoria de que, embora a terapia anti-PD1 possa ser um tratamento efetivo de MCM em alguns pacientes, o cérebro ainda pode representar um local de falha em um subgrupo de pacientes. ${ }^{(35,36)}$ Esse achado é corroborado por dados do 
estudo COMBI-MB, em que $53 \%$ dos pacientes que receberam dabrafenibe e trametinibe tiveram progressão somente no SNC. ${ }^{(21)}$ Os dados do CheckMate-204 ainda estão em fase de maturação, mas a PFS intracraniana em 9 meses foi de 59,5\%, comparado a $70,4 \%$ para a PFS extracraniana. ${ }^{(28)} \mathrm{O}$ tempo de seguimento ainda é essencial para tirar quaisquer conclusões sobre como a doença do SNC se comportará em comparação com a doença extracraniana nesta coorte prospectiva. É importante ressaltar que a progressão apenas do SNC foi associada à melhora de SG em comparação com pacientes com progressão sistêmica e do SNC concomitantes no nosso trabalho. Esse resultado foi consistente nos grupos "Com MCM Prévia" e "Sem MCM Prévia". A aquisição de imagens radiológicas de SNC deve, portanto, ser obtida de maneira rotineira durante todo o curso de um paciente com melanoma metastático, independentemente da presença de MCM prévia ou resposta sistêmica ao tratamento.

A radionecrose, uma complicação conhecida da radioterapia ao SNC, representa um desafio peculiar para essa população. Embora se desenvolva em uma minoria de pacientes, afetou $11,3 \%$ dos pacientes que receberam radiocirurgia estereotáxica no grupo "Com MCM Prévia", com a maior parte deles requerendo o uso prolongado de corticosteróides. Apesar de não desenvolver qualquer progressão sistêmica ou da doença intracraniana, alguns desses pacientes necessitaram de intervenção neurocirúrgica adicional. Se o uso de anti-PD1 eleva o risco de RN naqueles indivíduos que recebem radioterapia cerebral permanece controverso. Taxas de RN de até $37 \%$ foram relatadas em outros estudos menores com populações similares de pacientes, assegurando-se então a necessidade de conscientização sobre essa potencial complicação de imunoterapia combinada com a radiação. ${ }^{(37-41)}$ No entanto, uma grande colaboração multi-institucional recente encontrou uma incidência de $17 \%$ de RN associada à terapia com anti-PD-1, um número mais próximo do demonstrado em nossa coorte. ${ }^{(42)}$ Embora um tempo de seguimento maior possa ser necessário para o desenvolvimento de $\mathrm{RN}$, o tempo mediano até sua instalação no presente estudo foi curto (5,5 meses) a partir do início do anti-PD-1.

Apesar de ter uma biologia diferente, incluímos 18 pacientes com melanoma uveal nesta coorte. Devido à sua raridade e falta de tropismo para o cérebro (apenas $5-15 \%$ dos tumores avançados metastatizam para o cérebro) há uma escassez de dados sobre como o anti-PD-1 pode afetar a nova incidência de MCM nesta população, particularmente devido à baixa atividade de anti-PD-1 como agente único. ${ }^{(43,44)}$ Descobrimos que 4 deles desenvolveram MCM, três dos quais 
desenvolveram MCM após exposição a anti-PD-1. Embora nenhuma conclusão formal possa ser formada devido ao pequeno número de pacientes, a impressão é de que o anti-PD1 pode não trazer benefícios no que diz respeito à prevenção do desenvolvimento de MCM nesta população em particular.

O nosso estudo apresenta algumas limitações. A sua natureza retrospectiva, e o uso de uma coorte de pacientes de um único centro, com a maioria deles tratados fora de um ensaio clínico, podem trazer dificuldades de registro apropriado de dados. Ao mesmo tempo, porém, representa o maior estudo até o momento de dados do mundo real. Outra limitação importante é de que as taxas de resposta intracraniana ao anti-PD-1, um assunto de grande interesse atual, não puderam ser avaliadas devido à ampla maioria dos pacientes ter recebido terapia local dirigida ao SNC antes do início do anti-PD-1, e após a progressão. Restringimos também nossa coorte para pacientes recebendo anti-PD-1 isolado. À época do desenho do estudo, o papel fundamental da combinação com anti-CTLA-4 não era conhecido para metástases cerebrais. Por fim, dados de doença sistêmica para o grupo "Sem MCM Prévia” que não desenvolveu MCM não puderam ser obtidos.

Encontramos uma incidência de 27,3\% de MCM após a exposição a anti-PD-1 (para pacientes sem diagnóstico prévio de MCM), embora apenas 8,6\% tenham sido encontrados durante a terapia anti-PD-1 ou em até 30 dias após a interrupção. A potencial morbidade de MCM não detectada, a possibilidade de uso precoce de radioterapia estereotáxica e o fato do diagnóstico ou progressão de MCM poderem ocorrer sem progressão sistêmica concomitante reforçam a importância da vigilância contínua com imagens radiológicas durante a terapia com anti-PD-1. O desenvolvimento de MCM continua sendo um forte preditor de morte, apesar dos avanços na terapia sistêmica e local do SNC. Mais estudos de moléculas especificamente para a população com MCM ativa são necessários para minimizar os danos do melanoma a esse grupo de mau prognóstico. 


\section{CONCLUSÕES}

1. A incidência de novas metástases cerebrais por melanoma em pacientes sem diagnóstico de doença intracraniana antes do início de anti-PD-1 foi de $27,3 \%$;

2. Em quase metade dos casos onde houve progressão intracraniana, a doença extracraniana se encontrava estável, sugerindo que o sistema nervoso central ainda pode representar um santuário de doença. A percentagem de pacientes que não apresentaram progressão em nenhum sítio, porém, foi semelhante nos grupos com e sem metástases cerebrais pré-existentes;

3. A sobrevida global a partir do início do anti-PD-1 não foi significativamente diferente para pacientes sem metástase cerebral por melanoma prévia ao uso de anti-PD-1 em comparação com pacientes com metástase cerebral por melanoma prévia. Porém, desenvolver metástase cerebral por melanoma durante ou após o anti-PD-1 foi associado com pior sobrevida global. 


\section{APRESENTAÇÃO DO ARTIGO}

Schvartsman G, Ma J, Bassett RL Jr, Haydu LE, Amaria RN, Hwu P, et al. Incidence, patterns of progression, and outcomes of preexisting and newly discovered brain metastases during treatment with anti-PD-1 in patients with metastatic melanoma. Cancer. 2019. doi: 10.1002/cncr.32454. [Epub ahead of print]. 


\section{MÉTRICAS}

\subsection{Métricas do periódico}

Métricas do periódico nos últimos cinco anos.

\subsubsection{Fator de impacto}

Periódico: Cancer

ISSN: 0008-543X

Fator de impacto 2018: 6.102

\subsection{2}

2018 Journal Impact Factor

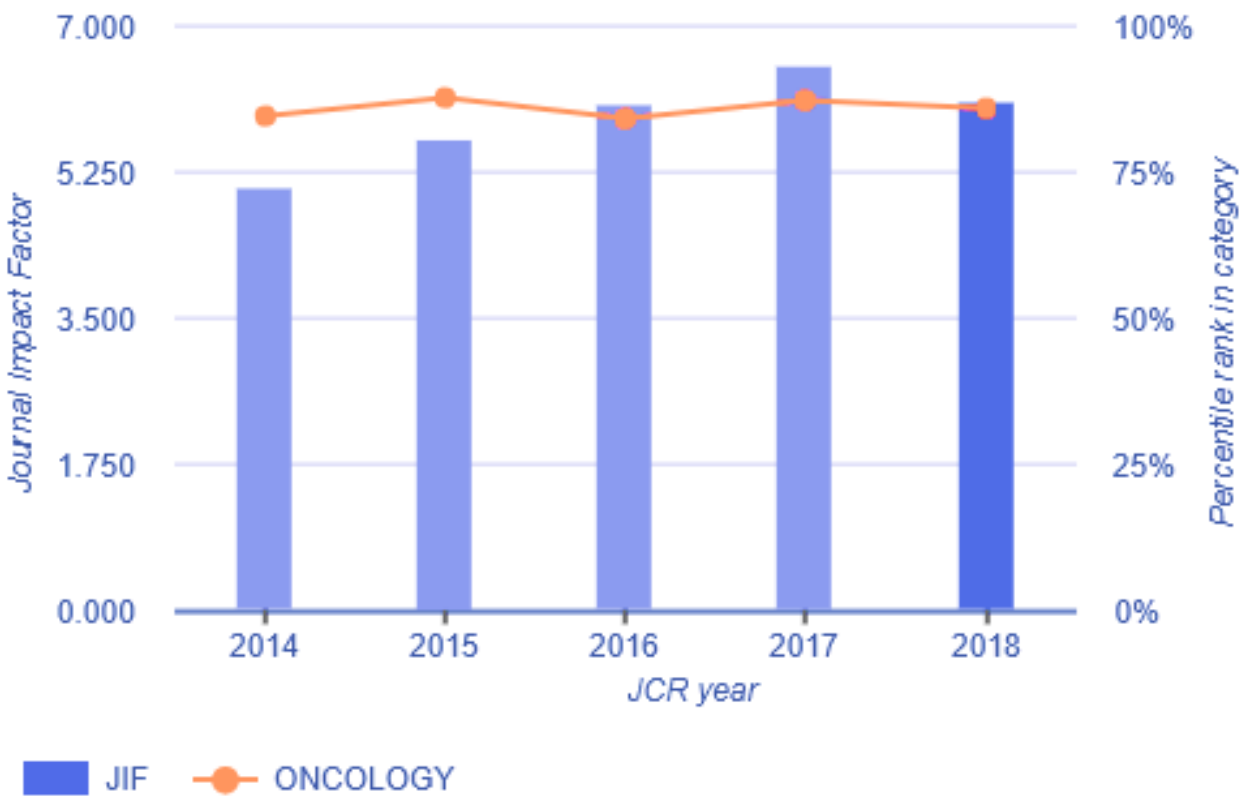

Fonte: Journal Citation Reports 


\subsection{Métricas do artigo}

\subsubsection{Citações}

Não foram localizadas citações para o artigo nas bases de dados PubMed, Scopus, Embase e Web of Science.

Avaliação realizada em 15 de outubro de 2019.

\subsubsection{Altmetria}

Dados altmétricos coletados em 15 de outubro de 2019.

\section{Escore}

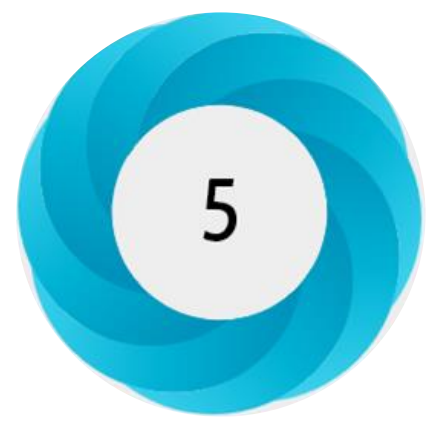

Variáveis

Menções em rede

Twitter

9

Origem das menções

Estados Unidos

3

Austrália

1

Brasil

1

Portugal

1

Desconhecida 9 3

Quantidade

\subsection{Métricas do autor}

\subsubsection{Redes científicas}

Participação do autor nas redes científicas e seus respectivos endereços eletrônicos, onde o autor registra e disponibiliza suas publicações. 
Rede científica

ORCID

Lattes

ScopusID

Publons/ResearcherID

Academia.edu
Link para acesso ao perfil

https://orcid.org/0000-0001-6601-3726

http://lattes.cnpq.br/8339902046540036

https://www.scopus.com/authid/detail.uri?authorld=55246851400

https://publons.com/researcher/2011919/gustavo-schvartsman/

https://independent.academia.edu/GustavoSchvartsman

\subsubsection{Trabalhos publicados}

Trabalhos publicados e indexados na base de dados referencial

Web of Science.

\begin{tabular}{|c|c|c|c|c|}
\hline \multicolumn{5}{|c|}{ Publicações } \\
\hline Ano & $\mathbf{F I}$ & Categoria & Referência & Citações \\
\hline 2012 & 0.626 & Article & $\begin{array}{l}\text { Goldenberg A, Rivas CE, Schvartsman } \\
\text { G. May titanium spiral tacks contribute to } \\
\text { intra-abdominal adhesion formation? } \\
\text { Acta Cir Bras. } 2012 ; 27(6): 430-2 \text {. }\end{array}$ & 0 \\
\hline 2016 & 6.294 & Review & $\begin{array}{l}\text { Schvartsman G, Ferrarotto R, } \\
\text { Massarelli E. Checkpoint inhibitors in } \\
\text { lung cancer: latest developments and } \\
\text { clinical potential. Ther Adv Med Oncol. } \\
2016 ; 8(6): 460-73 \text {. Review. }\end{array}$ & 17 \\
\hline 2016 & 2.608 & Case Report & $\begin{array}{l}\text { Schvartsman G, Wagner MJ, Zobniw } \\
\text { CM, Trinh VA, Patel S, Somaiah N. An } \\
\text { unusual case of central retinal vein } \\
\text { occlusion and review of the toxicity } \\
\text { profile of regorafenib in GIST patients. } \\
\text { Curr Oncol Rep. } 2016 ; 18(8): 49 \text {. Review. }\end{array}$ & 0 \\
\hline 2017 & 4.486 & Article & $\begin{array}{l}\text { Schvartsman G, Peng SA, Bis G, Lee } \\
\text { JJ, Benveniste MF, Zhang J, et al. } \\
\text { Response rates to single-agent } \\
\text { chemotherapy after exposure to immune } \\
\text { checkpoint inhibitors in advanced non- } \\
\text { small cell lung cancer. Lung Cancer. } \\
2017 ; 112: 90-5 \text {. }\end{array}$ & 32 \\
\hline 2017 & 19.384 & Letter & $\begin{array}{l}\text { Schvartsman G, Perez K, Sood G, } \\
\text { Katkhuda R, Tawbi H. Immune } \\
\text { checkpoint inhibitor therapy in a liver } \\
\text { transplant recipient with melanoma. Ann } \\
\text { Intern Med. } 2017 ; 167(5): 361-2 \text {. }\end{array}$ & 11 \\
\hline 2017 & 8.374 & Case Report & $\begin{array}{l}\text { Schvartsman G, Perez K, Flynn JE, } \\
\text { Myers JN, Tawbi H. Safe and effective } \\
\text { administration of T-VEC in a patient with } \\
\text { heart transplantation and recurrent } \\
\text { locally advanced melanoma. J } \\
\text { Immunother Cancer. 2017;5:45. }\end{array}$ & 10 \\
\hline
\end{tabular}




\begin{tabular}{|c|c|c|c|c|}
\hline 2017 & 3.202 & Article & $\begin{array}{l}\text { Schvartsman G, Gutierrez-Barrera AM, } \\
\text { Song J, Ueno NT, Peterson SK, Arun B. } \\
\text { Association between weight gain during } \\
\text { adjuvant chemotherapy for early-stage } \\
\text { breast cancer and survival outcomes. } \\
\text { Cancer Med. } 2017 ; 6(11): 2515-22 \text {. }\end{array}$ & 5 \\
\hline 2017 & 4.122 & Article & $\begin{array}{l}\text { Schvartsman G, Wagner MJ, Amini B, } \\
\text { Zobniw CM, Trinh VA, Barbo AG, et al. } \\
\text { Treatment patterns, efficacy and toxicity } \\
\text { of regorafenib in gastrointestinal stromal } \\
\text { tumour patients. Sci Rep. } \\
2017 ; 7(1): 9519 \text {. }\end{array}$ & 4 \\
\hline 2017 & 3.249 & Article & $\begin{array}{l}\text { Schvartsman G, Park M, Liu DD, Yennu } \\
\text { S, Bruera E, Hui D. Could objective tests } \\
\text { be used to measure fatigue in patients } \\
\text { with advanced cancer? J Pain Symptom } \\
\text { Manage. 2017;54(2):237-44. }\end{array}$ & 4 \\
\hline 2018 & 14.196 & Article & $\begin{array}{l}\text { Glitza Oliva IC, Schvartsman G, Tawbi } \\
\text { H. Advances in the systemic treatment of } \\
\text { melanoma brain metastases. Ann Oncol. } \\
2018 ; 29(7): 1509-20 \text {. }\end{array}$ & 4 \\
\hline 2018 & 3.471 & Review & $\begin{array}{l}\text { Rocha FB, Falcone AB, Buzaid AC, } \\
\text { Pimenta JM, Schvartsman G, Frasson } \\
\text { AL. Neoadjuvant therapy for breast } \\
\text { cancer treatment: an expert panel } \\
\text { recommendation from the Brazilian } \\
\text { Society of Breast Surgeons 2018. Breast } \\
\text { Cancer Res Treat. 2018;172(2):265-72. } \\
\text { Review. }\end{array}$ & 2 \\
\hline 2019 & 2.442 & Review & $\begin{array}{l}\text { Schvartsman G, Pinto NA, Bell D, } \\
\text { Ferrarotto R. Salivary gland tumors: } \\
\text { molecular characterization and } \\
\text { therapeutic advances for metastatic } \\
\text { disease. Head } \\
\text { Neck. 2019;41(1):239-47. Review. }\end{array}$ & 1 \\
\hline 2019 & 6.102 & Article & $\begin{array}{l}\text { Schvartsman G, Ma J, Bassett RL Jr, } \\
\text { Haydu LE, Amaria RN, Hwu P, et al. } \\
\text { Incidence, patterns of progression, and } \\
\text { outcomes of preexisting and newly } \\
\text { discovered brain metastases during } \\
\text { treatment with anti-PD-1 in patients with } \\
\text { metastatic melanoma. Cancer. } 2019 \text {. doi: } \\
10.1002 / \text { cncr.32454. [Epub ahead of } \\
\text { print]. }\end{array}$ & 0 \\
\hline 2019 & 2.450 & Article & $\begin{array}{l}\text { Schvartsman G, Carneiro AP, Filippi } \\
\text { RZ, Rao P, Msaouel P. Rapid deep } \\
\text { responses with nivolumab plus } \\
\text { ipilimumab in papillary renal cell } \\
\text { carcinoma with sarcomatoid } \\
\text { dedifferentiation. Clin Genitourin Cancer. } \\
2019 ; 17(4): 315-8 \text {. }\end{array}$ & 0 \\
\hline 2019 & 5.670 & Review & $\begin{array}{l}\text { Schvartsman G, Taranto P, Glitza IC, } \\
\text { Agarwala SS, Atkins MB, Buzaid AC. } \\
\text { Management of metastatic cutaneous } \\
\text { melanoma: updates in clinical practice. }\end{array}$ & 0 \\
\hline
\end{tabular}


Ther Adv Med Oncol.

2019;11:1758835919851663. Review.

Schvartsman G, Kaliks RA. Clinical and

$2019 \quad 70.670 \quad$ Letter genomic risk in adjuvant therapy for breast cancer. N Engl J Med.

\subsubsection{Mediana do fator de impacto dos trabalhos publicados}

\begin{tabular}{|c|c|}
\hline Fator de impacto das publicações & \multirow{}{}{} \\
\hline 0.626 \\
\hline 2.442 \\
\hline 2.450 \\
\hline 2.608 \\
\hline 3.202 \\
\hline 3.249 \\
\hline 3.471 \\
\hline 4.122 \\
\hline 4.486 \\
\hline 5.670 \\
\hline 6.102 \\
\hline 6.294 \\
\hline 8.374 \\
\hline 14.196 \\
\hline 19.384 \\
\hline 70.670 \\
\hline
\end{tabular}

\subsubsection{Citações recebidas}

\begin{tabular}{|ccc|}
\hline \multicolumn{3}{|c|}{ Publicações $\mathbf{x}$ Citações } \\
\hline Ano & Publicações & Citações \\
\hline 2012 & 1 & 0 \\
\hline 2016 & 2 & 17 \\
\hline 2017 & 6 & 66 \\
\hline 2018 & 2 & 6 \\
\hline 2019 & 5 & 1 \\
\hline Total & $\mathbf{1 6}$ & $\mathbf{9 0}$ \\
\hline
\end{tabular}




\subsection{5 h-index e m-quociente}

\begin{tabular}{|lc|}
\hline \multicolumn{1}{|c|}{ Cálculo do h-index } \\
\hline \multicolumn{1}{|c|}{ Tópicos } & Dados \\
\hline Quantidade de publicações: & 16 \\
\hline Soma do número de citações: & 90 \\
\hline Artigos que receberam citações: & 10 \\
\hline Média de citações por item: & 5,6 \\
\hline h-index: & $\mathbf{5}$ \\
\hline
\end{tabular}

Cálculo do m-quociente

\begin{tabular}{|l|c|c|}
\hline \multicolumn{2}{|c|}{ Caiculo do m-quociente } \\
\hline h-index & 5 & \multirow{2}{*}{$\mathbf{0 , 7}$} \\
\hline Número de anos de publicação & 7 & \\
\hline
\end{tabular}

\begin{tabular}{|c|c|c|c|c|}
\hline \multicolumn{5}{|c|}{ Publicações - h-index } \\
\hline Ano & $\mathbf{F I}$ & Categoria & Referência & Citações \\
\hline 2017 & 4.486 & Article & $\begin{array}{l}\text { Schvartsman G, Peng SA, Bis G, Lee JJ, } \\
\text { Benveniste MF, Zhang J, et al. Response rates to } \\
\text { single-agent chemotherapy after exposure to } \\
\text { immune checkpoint inhibitors in advanced non- } \\
\text { small cell lung cancer. Lung Cancer. } \\
2017 ; 112: 90-5 \text {. }\end{array}$ & 32 \\
\hline 2016 & 6.294 & Review & $\begin{array}{l}\text { Schvartsman G, Ferrarotto R, Massarelli E. } \\
\text { Checkpoint inhibitors in lung cancer: latest } \\
\text { developments and clinical potential. Ther Adv } \\
\text { Med Oncol. 2016;8(6):460-73. Review. }\end{array}$ & 17 \\
\hline 2017 & 19.384 & Letter & $\begin{array}{l}\text { Schvartsman G, Perez K, Sood G, Katkhuda R, } \\
\text { Tawbi H. Immune checkpoint inhibitor therapy in } \\
\text { a liver transplant recipient with melanoma. Ann } \\
\text { Intern Med. } 2017 ; 167(5): 361-2 \text {. }\end{array}$ & 11 \\
\hline 2017 & 8.374 & $\begin{array}{l}\text { Case } \\
\text { Report }\end{array}$ & $\begin{array}{l}\text { Schvartsman G, Perez K, Flynn JE, Myers JN, } \\
\text { Tawbi H. Safe and effective administration of T- } \\
\text { VEC in a patient with heart transplantation and } \\
\text { recurrent locally advanced melanoma. J } \\
\text { Immunother Cancer. } 2017 ; 5: 45 \text {. }\end{array}$ & 10 \\
\hline 2017 & 3.202 & Article & $\begin{array}{l}\text { Schvartsman G, Gutierrez-Barrera AM, Song J, } \\
\text { Ueno NT, Peterson SK, Arun B. Association } \\
\text { between weight gain during adjuvant } \\
\text { chemotherapy for early-stage breast cancer and } \\
\text { survival outcomes. Cancer Med. } \\
2017 ; 6(11): 2515-22 \text {. }\end{array}$ & 5 \\
\hline
\end{tabular}




\begin{tabular}{|c|c|c|c|c|}
\hline 2017 & 4.122 & Article & $\begin{array}{l}\text { Schvartsman G, Wagner MJ, Amini B, Zobniw } \\
\text { CM, Trinh VA, Barbo AG, et al. Treatment } \\
\text { patterns, efficacy and toxicity of regorafenib in } \\
\text { gastrointestinal stromal tumour patients. Sci Rep. } \\
2017 ; 7(1): 9519 \text {. }\end{array}$ & 4 \\
\hline 2017 & 3.249 & Article & $\begin{array}{l}\text { Schvartsman G, Park M, Liu DD, Yennu S, } \\
\text { Bruera E, Hui D. Could objective tests be used to } \\
\text { measure fatigue in patients with advanced } \\
\text { cancer? J Pain Symptom Manage. } \\
2017 ; 54(2): 237-44 \text {. }\end{array}$ & 4 \\
\hline 2018 & 14.196 & Article & $\begin{array}{l}\text { Glitza Oliva IC, Schvartsman G, Tawbi H. } \\
\text { Advances in the systemic treatment of melanoma } \\
\text { brain metastases. Ann Oncol. 2018;29(7):1509- } \\
20 \text {. }\end{array}$ & 4 \\
\hline 2018 & 3.471 & Review & $\begin{array}{l}\text { Rocha FB, Falcone AB, Buzaid AC, Pimenta JM, } \\
\text { Schvartsman G, Frasson AL. Neoadjuvant } \\
\text { therapy for breast cancer treatment: an expert } \\
\text { panel recommendation from the Brazilian Society } \\
\text { of Breast Surgeons 2018. Breast Cancer Res } \\
\text { Treat. 2018;172(2):265-72. Review. }\end{array}$ & 2 \\
\hline 2019 & 2.442 & Review & $\begin{array}{l}\text { Schvartsman G, Pinto NA, Bell D, Ferrarotto R. } \\
\text { Salivary gland tumors: Molecular characterization } \\
\text { and therapeutic advances for metastatic disease. } \\
\text { Head Neck. } 2019 ; 41(1): 239-47 \text {. Review. }\end{array}$ & 1 \\
\hline 2012 & 0.626 & Article & $\begin{array}{l}\text { Goldenberg A, Rivas CE, Schvartsman G. May } \\
\text { titanium spiral tacks contribute to intra-abdominal } \\
\text { adhesion formation? Acta Cir Bras. } \\
2012 ; 27(6): 430-2 \text {. }\end{array}$ & 0 \\
\hline 2016 & 2.608 & $\begin{array}{l}\text { Case } \\
\text { Report }\end{array}$ & $\begin{array}{l}\text { Schvartsman G, Wagner MJ, Zobniw CM, Trinh } \\
\text { VA, Patel S, Somaiah N. An unusual case of } \\
\text { central retinal vein occlusion and review of the } \\
\text { toxicity profile of regorafenib in GIST patients. } \\
\text { Curr Oncol Rep. } 2016 ; 18(8): 49 \text {. Review. }\end{array}$ & 0 \\
\hline 2019 & 6.102 & Article & $\begin{array}{l}\text { Schvartsman G, Ma J, Bassett RL Jr, Haydu LE, } \\
\text { Amaria RN, Hwu P, et al. Incidence, patterns of } \\
\text { progression, and outcomes of preexisting and } \\
\text { newly discovered brain metastases during } \\
\text { treatment with anti-PD-1 in patients with } \\
\text { metastatic melanoma. Cancer. } 2019 \text {. doi: } \\
\text { 10.1002/cncr.32454. [Epub ahead of print]. }\end{array}$ & 0 \\
\hline 2019 & 2.450 & Article & $\begin{array}{l}\text { Schvartsman G, Carneiro AP, Filippi RZ, Rao P, } \\
\text { Msaouel P. Rapid deep responses with } \\
\text { nivolumab plus ipilimumab in papillary renal cell } \\
\text { carcinoma with sarcomatoid dedifferentiation. Clin } \\
\text { Genitourin Cancer. } 2019 ; 17(4): 315-8 \text {. }\end{array}$ & 0 \\
\hline 2019 & 5.670 & Review & $\begin{array}{l}\text { Schvartsman G, Taranto P, Glitza IC, Agarwala } \\
\text { SS, Atkins MB, Buzaid AC. Management of } \\
\text { metastatic cutaneous melanoma: updates in } \\
\text { clinical practice. Ther Adv Med Oncol. } \\
\text { 2019;11:1758835919851663. Review. }\end{array}$ & 0 \\
\hline 2019 & 70.670 & Letter & $\begin{array}{l}\text { Schvartsman G, Kaliks RA. Clinical and genomic } \\
\text { risk in adjuvant therapy for breast cancer. N Engl } \\
\text { J Med. } 2019 ; 381(13): 1289-91 \text {. }\end{array}$ & 0 \\
\hline
\end{tabular}




\section{REFERÊNCIAS}

1. Narayanan DL, Saladi RN, Fox JL. Ultraviolet radiation and skin cancer. Int J Dermatol. 2010;49(9):978-86.

2. Miller KD, Nogueira L, Mariotto AB, Rowland JH, Yabroff KR, Alfano CM, et al. Cancer treatment and survivorship statistics, 2019. CA Cancer J Clin. 2019;69(5):363-85.

3. Brasil. Ministério da Saúde. Instituto Nacional de Câncer. Câncer de pele melanoma [Internet]. Rio de Janeiro: INCA [atualizado em 2019 nov 23; citado em 2019 ago 15]. Disponível em: https://www.inca.gov.br/tipos-de-cancer/cancer-de-pele-melanoma.

4. Schvartsman G, Taranto P, Glitza IC, Agarwala SS, Atkins MB, Buzaid AC. Management of metastatic cutaneous melanoma: updates in clinical practice. Ther Adv Med Oncol. 2019;11:1758835919851663.

5. Flaherty KT, Puzanov I, Kim KB, Ribas A, McArthur GA, Sosman JA, et al. Inhibition of mutated, activated BRAF in metastatic melanoma. N Engl J Med. 2010;363(9):809-19.

6. Robert C, Grob JJ, Stroyakovskiy D, Karaszewska B, Hauschild A, Levchenko E, et al. Fiveyear outcomes with dabrafenib plus trametinib in metastatic melanoma. $\mathrm{N}$ Engl J Med. 2019;381(7):626-36.

7. Okazaki T, Honjo T. PD-1 and PD-1 ligands: from discovery to clinical application. Int Immunol. 2007;19(7):813-24.

8. Riaz N, Havel JJ, Makarov V, Desrichard A, Urba WJ, Sims JS, et al. Tumor and microenvironment evolution during immunotherapy with nivolumab. Cell. 2017;171(4):93449.e16.

9. Schadendorf D, Hodi FS, Robert C, Weber JS, Margolin K, Hamid O, et al. Pooled analysis of long-term survival data from phase II and phase III trials of ipilimumab in unresectable or metastatic melanoma. J Clin Oncol. 2015;33(17):1889-94.

10. Wolchok JD, Neyns B, Linette G, Negrier S, Lutzky J, Thomas L, et al. Ipilimumab monotherapy in patients with pretreated advanced melanoma: a randomised, double-blind, multicentre, phase 2, dose-ranging study. Lancet Oncol. 2010;11(2):155-64.

11. Hamid O, Robert C, Daud A, Hodi FS, Hwu WJ, Kefford R, ET AL. Five-year survival outcomes for patients with advanced melanoma treated with pembrolizumab in KEYNOTE-001. Ann Oncol. 2019;30(4):582-8.

12. Hodi FS, Chiarion-Sileni V, Gonzalez R, Grob JJ, Rutkowski P, Cowey CL, et al. Nivolumab plus ipilimumab or nivolumab alone versus ipilimumab alone in advanced melanoma (CheckMate 067): 4-year outcomes of a multicentre, randomised, phase 3 trial. Lancet Oncol. 2018;19(11):1480-92.

13. Nayak L, Lee EQ, Wen PY. Epidemiology of brain metastases. Curr Oncol Rep. 2012;14(1):48-54.

14. Patel JK, Didolkar MS, Pickren JW, Moore RH. Metastatic pattern of malignant melanoma. A study of 216 autopsy cases. Am J Surg. 1978;135(6):807-10. 
15. de la Monte SM, Moore GW, Hutchins GM. Patterned distribution of metastases from malignant melanoma in humans. Cancer Res. 1983;43(7):3427-33.

16. Fife KM, Colman MH, Stevens GN, Firth IC, Moon D, Shannon KF, et al. Determinants of outcome in melanoma patients with cerebral metastases. J Clin Oncol. 2004;22(7):1293-300.

17. Agarwala SS, Kirkwood JM, Gore M, Dreno B, Thatcher N, Czarnetski B, et al.

Temozolomide for the treatment of brain metastases associated with metastatic melanoma: a phase II study. J Clin Oncol. 2004;22(11):2101-7.

18. Dummer R, Goldinger SM, Turtschi CP, Eggmann NB, Michielin O, Mitchell L, et al. Vemurafenib in patients with BRAF(V600) mutation-positive melanoma with symptomatic brain metastases: final results of an open-label pilot study. Eur J Cancer. 2014;50(3):611-21.

19. McArthur GA, Maio M, Arance A, Nathan P, Blank C, Avril MF, et al. Vemurafenib in metastatic melanoma patients with brain metastases: an open-label, single-arm, phase 2 , multicentre study. Ann Oncol. 2017;28(3):634-41.

20. Long GV, Trefzer U, Davies MA, Kefford RF, Ascierto PA, Chapman PB, et al. Dabrafenib in patients with Val600Glu or Val600Lys BRAF-mutant melanoma metastatic to the brain (BREAKMB): a multicentre, open-label, phase 2 trial. Lancet Oncol. 2012;13(11):1087-95.

21. Davies MA, Saiag P, Robert C, Grob JJ, Flaherty KT, Arance A, et al. Dabrafenib plus trametinib in patients with $\mathrm{BRAF}^{\mathrm{V} 600}$-mutant melanoma brain metastases (COMBI-MB): a multicentre, multicohort, open-label, phase 2 trial. Lancet Oncol. 2017;18(7):863-73.

22. Glitza Oliva IC, Schvartsman G, Tawbi H. Advances in the systemic treatment of melanoma brain metastases. Ann Oncol. 2018;29(7):1509-20.

23. Cruz-Orengo L, Holman DW, Dorsey D, Zhou L, Zhang P, Wright M, et al. CXCR7 influences leukocyte entry into the CNS parenchyma by controlling abluminal CXCL12 abundance during autoimmunity. J Exp Med. 2011;208(2):327-39.

24. Agrawal S, Anderson P, Durbeej M, van Rooijen N, Ivars F, Opdenakker G, et al. Dystroglycan is selectively cleaved at the parenchymal basement membrane at sites of leukocyte extravasation in experimental autoimmune encephalomyelitis. J Exp Med. 2006;203(4):1007-19.

25. Louveau A, Smirnov I, Keyes TJ, Eccles JD, Rouhani SJ, Peske JD, et al. Structural and functional features of central nervous system lymphatic vessels. Nature. 2015;523(7560):33741.

26. Margolin K, Ernstoff MS, Hamid O, Lawrence D, McDermott D, Puzanov I, et al. Ipilimumab in patients with melanoma and brain metastases: an open-label, phase 2 trial. Lancet Oncol. 2012;13(5):459-65.

27. Goldberg SB, Gettinger SN, Mahajan A, Chiang AC, Herbst RS, Sznol M, et al. Pembrolizumab for patients with melanoma or non-small-cell lung cancer and untreated brain metastases: early analysis of a non-randomised, open-label, phase 2 trial. Lancet Oncol. 2016;17(7):976-83.

28. Tawbi HA, Forsyth PA, Hodi FS, Lao CD, Moschos SJ, Hamid O, et al. Efficacy and safety of the combination of nivolumab (NIVO) plus ipilimumab (IPI) in patients with symptomatic melanoma brain metastases (CheckMate 204). J Clin Oncol. 2019,37(15 Suppl):9501. 
29. Long GV, Atkinson V, Lo S, Sandhu S, Guminski AD, Brown MP, et al. Combination nivolumab and ipilimumab or nivolumab alone in melanoma brain metastases: a multicentre randomised phase 2 study. Lancet Oncol. 2018;19(5):672-81.

30. Cohen JV, Tawbi H, Margolin KA, Amravadi R, Bosenberg M, Brastianos PK, et al. Melanoma central nervous system metastases: current approaches, challenges, and opportunities. Pigment Cell Melanoma Res. 2016;29(6):627-42.

31. Kohutek ZA, Yamada Y, Chan TA, Brennan CW, Tabar V, Gutin PH, et al. Long-term risk of radionecrosis and imaging changes after stereotactic radiosurgery for brain metastases. $\mathrm{J}$ Neurooncol. 2015;125(1):149-56.

32. Gummadi T, Zhang BY, Valpione S, Kim C, Kottschade LA, Mittapalli RK, et al. Impact of BRAF mutation and BRAF inhibition on melanoma brain metastases. Melanoma Res. 2015;25(1):75-9.

33. Peuvrel L, Saint-Jean M, Quéreux G, Brocard A, Khammari A, Knol AC, et al. Incidence and characteristics of melanoma brain metastases developing during treatment with vemurafenib. $J$ Neurooncol. 2014;120(1):147-54.

34. Robert C, Ribas A, Schachter J, Arance A, Grob JJ, Mortier L, et al. Pembrolizumab versus ipilimumab in advanced melanoma (KEYNOTE-006): post-hoc 5-year results from an openlabel, multicentre, randomised, controlled, phase 3 study. Lancet Oncol. 2019;20(9):1239-51.

35. Ransohoff RM, Engelhardt $B$. The anatomical and cellular basis of immune surveillance in the central nervous system. Nat Rev Immunol. 2012;12(9):623-35.

36. Wilson $\mathrm{EH}$, Weninger W, Hunter CA. Trafficking of immune cells in the central nervous system. J Clin Invest. 2010;120(5):1368-79.

37. Fang P, Jiang W, Allen P, Glitza I, Guha N, Hwu P, et al. Radiation necrosis with stereotactic radiosurgery combined with CTLA-4 blockade and PD-1 inhibition for treatment of intracranial disease in metastatic melanoma. J Neurooncol. 2017;133(3):595-602.

38. Kotecha R, Miller JA, Venur VA, Mohammadi AM, Chao ST, Suh JH, et al. Melanoma brain metastasis: the impact of stereotactic radiosurgery, BRAF mutational status, and targeted and/or immune-based therapies on treatment outcome. J Neurosurg. 2018;129(1):50-9.

39. Colaco RJ, Martin P, Kluger HM, Yu JB, Chiang VL. Does immunotherapy increase the rate of radiation necrosis after radiosurgical treatment of brain metastases? J Neurosurg. 2016;125(1):17-23.

40. Nardin C, Mateus C, Texier M, Lanoy E, Hibat-Allah S, Ammari S, et al. Tolerance and outcomes of stereotactic radiosurgery combined with anti-programmed cell death-1

(pembrolizumab) for melanoma brain metastases. Melanoma Res. 2018;28(2):111-9.

41. Tallet AV, Dhermain F, Le Rhun E, Noël G, Kirova YM. Combined irradiation and targeted therapy or immune checkpoint blockade in brain metastases: toxicities and efficacy. Ann Oncol. 2017;28(12):2962-76. Review.

42. da Silva IP, Glitza IC, Haydu LE, Johnpulle R, Banks PD, Grass GD, et al. Incidence, features and management of radionecrosis in melanoma patients treated with cerebral radiotherapy and anti-PD-1 antibodies. Pigment Cell Melanoma Res. 2019;32(4):553-63. 
43. Holfort SK, Lindegaard J, Isager P, Prause JU, Heegaard S. CNS metastasis from malignant uveal melanoma: a clinical and histopathological characterisation. Br J Ophthalmol. 2009;93(5):641-4.

44. Algazi AP, Tsai KK, Shoushtari AN, Munhoz RR, Eroglu Z, Piulats JM, et al. Clinical outcomes in metastatic uveal melanoma treated with PD-1 and PD-L1 antibodies. Cancer. 2016;122(21):3344-53. 


\section{Abstract}

Introduction: Melanoma brain metastases occur in up to $50 \%$ of patients with metastatic melanoma and represent a frequent site of systemic treatment failure. Immunotherapy has revolutionized the treatment of melanoma, and demonstrated efficacy in patients with melanoma brain metastases. However, little is known about the incidence of new melanoma brain metastases in undiagnosed patients, patterns of treatment progression and long-term outcomes in patients with and without melanoma brain metastases treated with anti-PD-1 immunotherapy. Purpose: To assess the incidence of melanoma brain metastases in patients without intracranial diagnosis prior to initiation of anti-PD-1 treatment; evaluate the patterns of progression of patients with and without melanoma brain metastases at the beginning of anti-PD-1 treatment; and to evaluate long-term outcomes in different populations in the current era of immunotherapy and intensive local therapy of brain metastases. Methods: 320 patients with metastatic melanoma treated with anti-PD-1 at MD Anderson Cancer Center were reviewed. Analyzes were performed to identify factors associated with brain metastasisfree survival and overall survival using Cox regression models and the Kaplan-Meier method. Results: The median age was 63.3 years. The incidence of new melanoma brain metastases among patients without prior diagnosis was $27.3 \%(\mathrm{~N}=67)$. Of these, $21(8.6 \%)$ developed melanoma brain metastases during anti-PD-1 treatment, of which $12(4.9 \%)$ showed central nervous system-only progression. Among patients with prior melanoma brain metastases, $15(20.0 \%)$ progressed only in the brain and $19(25.3 \%)$ progressed intra and extracranially; until data cutoff, 27 (36.0\%) had not progressed. Overall survival from initiation of anti-PD-1 was not significantly different for patients with or without prior melanoma brain metastases ( $P$-value $=0.359$ ), but developing melanoma brain metastases during or after anti-PD-1 was associated with poor overall survival (HR=4.70, 95\% Cl: 3.18-6.93). Radionecrosis occurred in $11.3 \%$ (7 of 62 ) of patients in the group with prior melanoma brain metastases who received stereotactic radiosurgery before anti-PD-1 start. Conclusions: Anti-PD-1 therapy may alter the natural history of patients with prior melanoma brain metastases, with no difference in outcomes for patients with or without melanoma brain metastases. However, anti-PD-1 failure in the central nervous system is frequent and still represents a poor prognostic factor. Our results support the activity of anti-PD-1 in melanoma brain metastases 
patients, but our findings suggest that routine brain imaging is recommended, even in the absence of neurological symptoms.

Keywords: Melanoma; Immune checkpoint inhibitors; Brain neoplasms/secondary; Treatment outcome 